\title{
Estudos sobre os flajelados parasitos dos mamiferos do Brazil pelo
}

\author{
Dr. Olympio Oliveira Ribeiro da Fonseca
}

(Com as estampas 1 e 2 e 4 figuras no texto.)

\section{Introdução.}

Ha algum tempo que empreendemos o estudo dos flajelados parasitos dos mamiferos, indijenas ou importados, existentes no Brazil. O resultado de nossas pesquizas constituiu assunto de duas notas prévias e de uma memoria já publicadas, esta ultima acompanhada de estudo geral dos flajelados.

A presente contribuição compreende tudo aquilo que de orijinal resultou de nosso trabalho sobre o assunto. Só nos ocupámos das protomonadinas parasitarias, porquanto os binucleados têm sido muito pesquizados e as rizomastijinas parasitarias não foram por nós encontradas.

Tornamos a descrever alguns parasitos, tendo utilizado para essa nova descrição apenas os elementos fornecidos pelos nossos preparados, do que resulta algumas delas parecerem deficientes, por não nos termos querido utilizar dos dados obtidos por outros autores; isso aconteceu, por exemplo, com as diversas especies de Giardia e de Trichomonas do homem, flajelados, aliaz, sobre cuja morfolojia ou distinção especifica reinam ainda muitas duvidas ou diverjencias. Tambem quanto aos hospedadores dos diversos para- sitos, só citamos observações por nós verificadas.

\section{Parte geral.}

\section{Historico.}

Os flajelados do intestino humano foram pela primeira vez verificados no Brazil em 1904, pelo Dr. OLYNTHO DE OLIVEIRA que encontrou no Rio Grande do Sul casos de disenteria com Giardia intestinalis (LAMBL) e Trichomonas hominis (DAVAINE). Os mesmos flajelados foram estudados, mais sob o ponto de vista medico que zoolojico, em 1912, pelo Dr. MELLO LEITÃO, no Rio de Janeiro.

BRUMPT, em 1913, em São Paulo, encontra Giardia intestinalis (LAMBL) que the parece rara naquela cidade.

Em 1014, os Drs. ARISTIDES MARQUES DA CUNHA e MAGARINOS TORRES observam em Lassance, estado de Minas Geraes, os primeiros casos brazileiros de colite com Chilomastix mesnili (WENYON).

Em 1915, o professor FICKER publica dados estatisticos sobre as disenterias reinantes em S. Paulo; refere ele ter observado 22 casos 
devidos flajelados intestinaes dos quaes $7 \mathrm{com}$ ocorrencia simultanea de amebas.

No mesmo ano, verificámos no Rio de Janeiro a presença de Trichomonas hominis (DAVAINE), T. vajinalis (LAMBL), Chilomastix mesnili (WENYON) e de um novo flajelado intestinal, Enteromonas hominis mihi, 1915.

Além dos flajelados intestinaes do homem, só nos constam sobre o assunto pesquizas recentes e ainda ineditas de CHAGAS sobre Cerodon rupestris WIED, o mocó.

Em 1915, publicámos a descrição de nova especie de Chilomastix, parasito do Mus (Epimys) norwegicus ERXL., o rato de esgoto.

\section{Material e tecnica.}

Realizámos 252 autopsias em diversos mamiferos ou observações in anima nobill. Foram por nós exanimadas 36 especies e 2 fórmas albinas de mamiferos pertencentes a faunas muito diversas; além deste material utilisámos, a titulo de comparação, o que obtivemos em muitas autopsias de aves, repteis, batraquios e insetos.

O material hnmano era constituido por urina ou fezes disentericas emitidas e levadas logo, contidas em vaso limpo e seco, a exame no laboratorio; o muco vajinal da mulher era colhido aseticamente $\mathrm{e}$ imediatamente exarninado.

O material de outros mamiferos era obtido nor autopsia em animal morto acidental ou propositadamente, ou ainda pela visita aos matadouros da cidade.

Após 0 imprecindivel exame a fresco, fixavamos pelo sublimado alcool de SCHAU. DINN o material ainda " humido, distendido em laminulas que, depois, eram coradas pelos proessos de HEIDENHAIN e de DOBELL. A's vezes, logo após a fixação, o preparado adquiria a côr rosea devida a reação do sublimado do fixador com derivados dos pigmentos biliares das fezes; outras vezes, após coloração a laminula se apresentava, em vez de intensamente corada em azul ou negro, com côr vermelha de barro; em um caso a imersão rapida e em outro pro- longada em alcool a 70 , bastava geralmente para corrijir aqueles defeitos do preparado.

Lista dos mamiferos por nós examinados e das respetivas protomonadinas parasitarias.

Ordo I. - BIMANA. (1)

I. Homo sapiens LINNEU, 1758. - Homem Chilomastix mesnili (WENYON, 1910).

Enteromonas hominis mihi, 1915. Giardia intestinalis (LAMBL., 1859).

Trichomonas hominis (DAVAINE, 1837.

Ordo II. - PRIMATES.

II. Cebus caray'á (HUMBOLDT, 1811). Macaco.

Giardia intestinalis (LAMBL., 1859)

III. Callithrix jacchus LINNEU, 1776.Sagui.

VI. Midas (Leontopithecus) rosalia LINNEU, 1776- Sagui vermelho.

Ordo IV. - CHIROPTERA.

V. Molossus obscurus GEOFFROY, 1805. - Morcego.

VI. * Nyctinomus gracilis (NATTERER) WAGNER, 1843. - Morcego.

VII. * Hemiderma perspicillatum (LINNEU, 1758). - Morcego.

VIII. * Glossophaga soricina (PALLAS, 1766). - Morcego.

IX. * Desmodus rufus WIED., 1826.Morcego.

(1) Seguimos na lista dos hospedadores o Catalogum Mammalium de Trouessart, tanto no que respeita á ordem seguida, como quanto aos nomes adotados. Excetuam-se desta regra, os nomes que estiverem assinalados por asterisco, os quaes corresponden aos maniferos, a nosso pedido determinados pelo Prof. Ali pio de Miranda Ribeiro, a quem deixamos aqui consignado nosso reconhecimento. 
Ordo VI.-CARNIVORA.

X. Ursus (Helarctos) malayanus RAFLES, 1822. -- Urso malaio.

XI Nasua rufa DESMAREST, 1820.Coati.

XII. Gallictis vittata SCHREBER, 1775.Furão.

X11I. Canis (Canis) familiaris LINNEU, 1758. - Cão.

XIV. Felis (Felis) domestica LINNEU, 1758. - Gato.

Ordo VIII. - RODENTIA.

$\mathrm{XV}$ a.Mus (Epimys) norwegicus ERXLEBEN 1777. - Rato dos esgotos. Chilomastix bittencourti mihi, 1915 Giardia muris (BENSEN, 1908). Octomitus muris (GRASSI, 1881). Trichomonas muris GALLI-VALERIO, 1907.

$\mathrm{XV}$ b. Mus (Epimys) norwegicus ERXLEBEN, 1777, fórira albina. - Rato branco.

Chilomastix bittencourti mihi, 1915 Octomitus muris (GRASSI, 1881) Trichomonas muris GALLI-VALERIO, 1907.

XVI. Mus (Epimys) rattus LINNEU, 1766-Rato dos tetos.

Octomitus muris (GRASSI, 1881).

XVII a. Mus (Mus) musculus LINNEU, 1776. - Camondongo cinzento.

Trichomonas muris GALLI-VALERIO, 1907.

XVII Mus (Mus) musculus LINNEU, 1766, fórma albina.-Camondongo branco.

Octomitus muris (GRASSI, 1881),

XVIII. Coendu prehensilis LINNEU, 1766. - Ouriço.

XIX Coendu villosus F. CUVIER, 1822.Ouriço.

Giardia cuniculi (BENSEN, 1908).

XX. Dasiprocta aguti LINNEU, 1776.Cotia.

Trichomastix caviae (GRASSI, 1881).
XXI. Agouti paca LJNNEU, 1766.

XXII. Cavia porcellus LINNEU, 1766.Cobaio.

Chilomatix intestinalis KUCZYNSKI, 1914.

Chilomitus cavia, nov. gen., nov. sp.

Sphoeromonas communis LIEBETANZ, 1910.

Trichomastix cavioe (GRASSI, 1881).

Trichomonas cavice DAVAINE, 1875.

XXIII. Cavia aperea ERXLEBEN, 1777.Preá.

Chilomitus cavia, nov. gen., nov. sp.

Trichomastıx caviae (GRASSI, 1881).

XXIV. Hydrocharus capybara LINNEU, 1766. - Capivára.

XXV. Oryctolagus cuniculus LINNEU, 1766. - Coelho.

Chilomastix cuniculi, nov. sp.

Giardia cuniculi (BENSEN, 1908)

Ordo X. - UNGULATA.

XXVI. Equus (Equus) caballus LINNEUCavalo.

XXVII. Sus (Sus) scrofa LINNEU, 1766.Porco.

XXVIII. Tayassus (Tayassus) tajacu LINNEU, 1766. - Caitetú.

XXIX. Cervus elaphus LINNEU, 1766.Veado europeu.

XXX. * Antilope cervicapra LINNEU.Antilope.

XXXI. Capra (Capra) hircus LINNEU, 1766. - Cabra.

Callimastix frontalis BRAUNE, 1914.

Chilomastix caproe, nov. sp.

Sphoromonas communis LIEBETANZ, 1910.

XXXII. Ovis aries LINNEU, 1766.Carneiro.

Callimastix frontalis BRAUNE, 1914.

XXXIII. Bos taurus IINNEU, 1766.Boi. 
Callimastix frontalis BRAUNE, 1914.

Sphoromonas communis LIEBETANZ, 1910.

Sphoromonas liebetanzi, nov. nom.

Ordo XIII. - EDENTATA.

XXXIV. Tatus (Tatus) novem-cinctus LINNEU, 1766.-Tatú.

Trichomonas tatusi, nov. sp.

Ordo XIV. - MARSUPIALIA.

XXXV. Macropus (Macropus) robustus GOULD, 1840. - Cangurú.

XXXVl. Didelphys (Didelphvs) marsupialis LINNEU, 1760. - Gambá.

\section{Biolojia geral.}

Pouca cousa de novo temos a dizer sobre a biolojia geral das protomonadinas parasitarias.

No estudo morfolojico, dous fatos se mostraram interessantes.

Encontrámos, não raro, em certos Trichomonas ( $T$. caviae, $T$. muris), um corpusculo ovoide, esferico ou com a forma de nexagono regular com angulos arredondados; nos preparados a fresco, no flajelado vivo, eles se mostravam refrinjentes; nos preparados tratados pela hematoxilina, esses corpusculos se mostraram intensamente corados em azul, nunca, porém, tão escuros quanto as formaçries cromaticas da celula; uma ou outra vez, pudemos vel-os livres no conteudo intestinal, provavelmente após destruição do parasito; não parece que seja substancia injerida porque no ponto em que ele existe é frequentemente substituido por um vacuolo na aparencia vasio. Talvez devamos colocar esse corpusculo no grupo das substancias cromatoides, com a volutina dos Trypanosoma.

Outro fato interessante, é a solução do problema da orijem e da natureza do axostilo. A nosso ver, a questão foi levantada sobre bases bastante falhas: foram comparadas conclusões diversas de autores que pesquizaram objetos diferentes.

No Trichomonas e nos tipos morfolo- jicos proximos desse genero (Trichomastix, Hexamastix), se encontra um bastonete espesso, rijido na aparencia tubular, vasio ou contendo granulações siderofilas esparsas, corta do muitas vezes em bisel numa ponta saliente para o exterior; a outra extremidade $\mathrm{e}$ interna $e$, ou se perde na massa plasmatica, ou se continua mais ou menos diretamente com o citostoma. Nunca vimos uma dessas formações apresentar conexão morfolojica com parte alguma do aparelho nucleo-flajelar. A's vezes, entretanto, este bastonete apresenta seus bordos limitados por duas linhas mais escuras em um ponto das quaes, junto ao biselamento terminal, podem existir duas granulações tambem escuras; é possivel que essas linhas e granulações sejam de natureza cromatica. Năo nos julgamos, entretanto, nem pelas nossas pequizas, nem pelos resultados discordantes obtidos por outros autores, autorizado a emitir qualquer hipotese sobre a natureza e a orijem desta formação, certamente de função esqueletica e a qual deve caber a designação de axostilo.

Em outros flajelados (Octomitus, Giardia), existem dois filamentos paralelos e independentes entre si, delgados, flexiveis, compactos, intensamente cromofilos, que partem de corpusculos basaes ligados aos nucleos por meio de rizoplastos, e, de outro lado, se continuam diretamente para o exterior com os flajelos. Sempre, nesses diplozoarios, a conexão morfolojica dos filamentos em questão com o aparelho nucleo-flajelar é patente, não deixando a menor duvida a qualquer observador atento. Muitos autores, entretanto, dão a esses filamentos a mesma designação de axostilo que deve cáber a formações totalmente diversas; daí a confusão reinante. Dc acordo com as conclusões !iradas por HARTMANN e CHAGAS, no estudo do filamento axial de Cercomonas parva, formação analoga aos filamentos da Giardia e do Octomitus, nos julgamos perfeitamente autorisado a atribuir a estes ultimos natureza cromatica e orijem nuclear indiscutiveis. ALEXEIEFF, estudando os Rhizomastix, encontrou um filamento analogo que denominou Rhizostylo e que caraterisou muito bem 
por esta frase já citada por KUCZYNSKI: "La fonction n'est pas analogue à celle de l'axostyle. En effet, ce dernier est surtout destiné à maintenir constante la forme du corps et représente ainsi une formation squelletique, tandis que Rhizostyle fait partie intégiante de l'appareil flagéllaire».

O caso da Giardia e do Octomitus é perfeitamente identico e, a admitirem-se esses dous diplozoarios como orijinados da fusão de dois Trichomastix, deve-se admitir que os flajelos posteriores destes forneceram os rizostilos e que seus axostilos devem ter desaparecido, por não existir formação a eles analoga em nenhum diplozoario parasito.

No ponto de vista da ecolojia geral, verificámos que tem muito maior importancia na escolha do habitat a localisação do flajelado no hospedador, do que a posição sistematica deste ultimo na escala zoolojica, assinı encontrámos Giardia dos mamiferos só no intestino delgado, onde não existem Trichomonas, ao passo que estes abundam no intestino grosso o qual é privadu de Giardia. A coabitação e a identidade de rejime alimentar favorecem a infeção, como tivemos ocasião de observar com cobaios que vieram a se infetar com Sphoromonas, parasito de ruminantes. Nenhum carnivoro se mostrou frequentrmente infetado e, dos herbivoros, eram os ruminantes e os roedores os que, quasi certamente, se mostravam parasitados.

Desse parasitismo, para os mamiferos em geral, não parece resultar ação patojenica; a inocuidade é a regra e, talvez, em certos casos o flajelado seja realmente util ao seu hospedador.

Os flajelados intestinaes do homem parecem constituir infeções secundarias consecutivas a quaesquer processos disenteriformes.

\section{Classificação das Protomonadinas. Generos parasitos.}

No sistema de HARTMAM e CHAGAS as protomonadinas são distribuidas em dous grupos, um deles compreende individuos asimetricos, tendo numero simples de organelas, e outro é constituido por flajelados providos de simetria bilateral e quasi sempre com numero duplo de todas as suas formações.

O primeiro grupo, Monozoa, inclue toda a antiga ordem das protomonadinas, com exclusão apenas dos binucleados, com os quaes HARTMANN constitue uma o dem á parte.

O segundo grupo, Diplozoa, corresponde ao antigo grupo dos Diplozoarios de DANGEARD e ás Distomata de KLEBS.

Embora isto não tenha sido expresso por HARTMANN e CHAGAS, julgamos dever considerar esses dous grupos com a categoria de sub-ordens.

Na sub-ordem Monozoa, HARTMANN inclue oito familias, numero este que aumentámos pela criação de uma nova familia.

A primeira familia, HARTMANN designa pelo nome de Cercomonadaca?; é caraterisada pela presença de um flajelo anterior que se continua com um filamento de orijem nuclear que atravessa o corpo e termina na extremidade posterior da celula, onde existe prolongamento caudal. Nesta familia, estaria incluido o genero Cercomonas. que outros julgam ser antes rizomastijina; foi por isso que LEMMERMANN propoz em 1913, a creação do genero Curcomastix, para incluir os protozoarios que HARTMANN e CHAGAS julgam Cercomonas; neste caso o nome da familia deveria ser mudado, de acordo com o nome do novo genero que lhe serviria de tipo. Não julgando, entretanto, que esteja resolvida a questão, preferimos seguir a opinião dos dous autores citados, conservando a designação por eles dada á familia, mudando apenas a desinencia, que, de acordo com as regras de nomenclatura zoolojica deve ser idae e não acece, terminação es ta adotada para os nomes de familias na nomenclatura botanica; esta mudança de desinencia será feita tambem para as outras familias da mesma ordem.

Colocamos na familia Cercomonadidoe, devido á presença de rizostilo, o genero Rhizomastix, creado por ALEXEIEFF em 1911, apezar de opiniões em contrario de outros, que preferem estudal-o na familia 
seguinte. A especie tipo deste genero é $R$. gracilis ALEXEIEFF, 1011.

A segunda familia, Oicomonadidoe se caraterisa por corpo globoso com flajelo unico e longo; encerra um só genero parasito, Sphoromonas, creado por LIEBETANZ em 1910 e cuja especie tipo é $S$. communis LIEBETANZ, 1910.

A' terceira familia, HARTMANN denomina Craspedomonadarece, designação que não póde prevalecer por não incluir ela nenhum genero de cujo nome pudesse este se orijinar; designaremos, por isso, a familia pelo nome adotado por POCHE, em 1913, de Codosigidce. Esta familia, caraterisada pela presença de um colarinho membranoso que cerca a base do flajelo, não encerra representante parasito.

A quarta familia que consideramos é a quinta de HARTMANN, Monadido, caraterisada pela presença de dous flajelos anteriores dos quaes um grande e um pezueno (Nebengeissel); nenhum representante parasito.

A nossa quinta familia é a sexta de HARTMANN, Amphimonadida, caraterisada pela presença de dous flajelos anteriore; de egual comprimento; nenhum representante parasito.

A sexta familia, na seriação que estabelecemos, é a quarta de HARTMANN, Bodonida, sobre cuja significação reinam as maiores diverjencias. $O$ genero que serve de tipo á familia é o genero Bodo, creado por EHRENBERG, em 1838, e cuja especie tipo por sua vez é $B$. saltans, descrita na mesma ocasião por aquele autor; até 1910 nenhuma duvida existia quanto aos protozoarios que deviam ser colocados no genero Bodo; naquele ano, porém, HARTMANN e CHAGAS descrevem novo genero de binucleados com o nome de Prowazekia e que difere do genero Bodo apenas por ter dous nucleos; temipos depois ALEXEIEFF verificou que $B$. saltans tambem tinha dous nucleos e era, portanto, binucleado, o que fazia cair em sinonimia o nome de Prowazekia. HARTMANN, porém, continua convencido da existencia autonoma do genero Prowazekia, por ter visto aqui no Brazil uma especie aquatica de Bodo, que ele não determinou e que não era binucleado. Para as especies parasitos, por alguns colocadas no genero Bodo, fôra creado o ganero Heteromita, nome que não póde prevalecer por já ter sido anteriormente empregado com significação diferente; por esse motivo, em 1912, ALEXEIEFF creou o genero Prowazekella, para a especie conhecida com o nome deBodo lacerto. Os representantes mononucleados do antigo genero Bodo, umá vez verificada a duplicidade nuclear da especie tipo deste genero, devem ser colccadas em novo genero que para tal fim ainda não foi creado.

A setima familia é Trimastigida, caraterisada pela presença de um flajelo anterior e de dous flajelos recorrentes; nenhum representante parasito.

A oitava familia é Tetramitido, cuja caraterisação era feita antigamente pela presença de quatro fiajelos; hoje, depois de conhecidos muitos flajelados proximos a tetramitidas tipicas, tendo numero diverso de flajelos, somos levado a caraterisar esta familia como: protomonadinas com quatro flajelos em dous grupos ou em um só grupo, podendo, então, um ser recorrente, ou ainda, com flajelos em numero diverso, mas tendo, ou membrana ondulante, ou flajelo rerorrente livre, ou axostılo, ou varias destas formações, simultaneamente. Esta familia compreende muitos generos e sub-generos de flajelados parasitos, segundo consta da lista seguinte:

Genero Chilomastix ALEXEIErF, 1910. Citnstoma com borda cromofila e percorrida por membrana ondulante, trez flajelos livres anteriores; não ha axostilo. Especie tipo: C. caulleryi (ALEXE!EFF, 1909).

Sub-genero Tetrachilomastix, nov. subgen. Difere do tipo precedente pela presença de quatro flajelos anteriores, em vez de trez. Especie tipo: Chilomastix (Tretrachilomastix) gallinarum MARTIN et ROBERTSON, 1911.

Genero Fanapepea PROWAZEK, 1911. Difere da Chilomastix por ter dous fiajelos anteriores, em vez de trez; sua existencia é duvidosa, por motivos que mais tarde estudaremos. Especie tipo: $F$. intestinalis PROWAZEK, 1911. 
Genero Cyathomastix PROWAZEK, 1914. Difere de Chilomastix pela presença de axostilo; existencia tambem duvidosa. Especie tipo: C. hominis, PROWAZEK, 1914.

Genero Difamıs GÄBEL, 1914. Difere de Chilomastix pela ausencia de membrana ondulante; existencia duvidosa. Especie tipo: D. tunensis GÄBEL, 1914.

Genero Embudononas MACKINNON, 1912. Difere de Chilomastix por ter apenas um flajelo anterior; encontrámos um representante deste genero em Stylopyga americana, a barata. Especie tipo: E. agilis MACKJNNON, 1912.

Genero Protrichomonas ALEXEIEFF, 1911. Trez flajelos anteriores, costa cromofila, axostilo não saliente para o exterior; não ha membrana ondulante, nem flajelo livre recorrente. Especie tipo: $P$. legeri ALEXEIEFF, 1911.

Genero Hexamastix ALEXEIEFF, 1912. Seis flajelos anteriores deseguaes, dos quaes um esboça membrana ondulante em seu inicio; axostilo saliente para o exterior; não existe costa. Especie tipo: $H$. batrachorum (ALEXEIEFF, 1911).

Genero Trichomonas DONNÉ, 1837. A membrana ondulante repousa sobre costa; trez flajelos anteriores, axostilo saliente para o exterior. Especie tipo: T. vaginalis DONNÉ, 1837.

Sub-genero Tretratrichomonas ALEXEIEFF, 1911. Difere do precedente por ter quatro flajelos anteriores, em vez de trez. Especie tipo: Trichomonas (Tetratrichomonas) prowazeki ALEXEIEFF, 1909.

Sub-genero Pentatrichomonas CHATTERJEE, 1915. Difere de Trichomonas pela presença de cinco flajelos anteriores. Especie tipo: Trichomonas (Pentatrichomonas) ardin delteili (DERIEU et RAYNAUD, 1914).

Genero Enteromonas, mihi, 1915. Um flajelo posterior e dous anteriores eguaes e mais curtos que o primeiro. Especie tipo: E. hominis, mihi, 1915.

Genero Trimitus ALEXEIEFF, 1910. Um flajelo posterior que atravessa o corpo e dous anteriores deseguaes e livres; existencia duvidosa, mesmo para o autor, que creou o genero com muitas reservas. Especie tipo: T. motelloe ALEXEIEFF, 1910.

Genero Trichomastix BLOCHMANN, 1884. Um flajelo recorrente livre, trez flajelus anteriores eguaes entre si, axostilo saliente para o exterior. Especie tipo: T. iacertoe (BÜTSCHLI, 1884).

Sub-genero Tetratrichomastix PARISI. Distingue-se do precedente por ter quatro flajelos anteriores em vez de trez. Especie tipo Trichomastix (Tetratrichomastix) orthopterorum PARISI, 1910.

Genero Chilomitus, mihi, 1915. Quatro flajelos anteriores eguaes, citostoma anterior, não ha axostilo. Especie tipo: C. cavia, mihi, 1915.

Genero Costia LECLERQ, 1890. Quatro flajelos anteriores, dous maiores e dous mensres, corpo asimetrico. Especie tipo: C. necatrix (HENNEGUY, 1884).

Genero Polymastix BÜTSC.HLI, 1884. Quatro flajelos anteriores eguaes, em dous grupos de dous cada um, axostilo não saliente para o exterior, periplasta rijido e estriado. Especie tipo: $P$. melolonthae BÜTSCHLI, 1884.

Genero Monocercomonas GRASSI, 1881. Quatro flajelos anteriores em dous grupos cada um deles com dous flajelos, dos quaes um ás vezes maior que o outro. Especie tipo: M. melolontce (GRASSI, 1879).

A familia que estabelecemos é Callimastigida, mihi, 1915, cujo unico genero foi creado por WEISSENBERG, em 1912, para a especie que denominou Callimastix cyclopis; julgava este autor que o parasito era proximo da familia Lophomonadidoe na qual, entretanto, não o fazia incluir ; como veremos pela discrição de $C$. frontalis, a qual concorda em suas linhas geraes, com a que WEISSENBERG fornece de C. cyclopis, o genero representa tipo perfeito de protomonadina, segundo a acepção atribuida a esse termo pelos atuaes representantes da escola de SCHAUDINN. No quadro das familias da ordem Protomonadina nenhuma existia em que licitamente se pudesse incluir o genero Callimastix; foram essas considerações que nos levaram a abandonar a insinuação de WEISSENBERG e a crear nova familia para 
este genero. A nova familia ficou assim caraterisada: protomonadinas arredondadas com flajelos numerosos em seu pólo anterior.

Genero Callimastix WEISSENBERG, 1912. Muitos flajelos delgados. longos, eguaes, anteriores e paralelos entre si, corpo globoso com periplasta muito rijido. Especie tipo: $C$. cyclopis WEISSENBERG, 1912.

Na sub-ordem Diplozoa, HARTMANN inclue uma unica familia a que denomina Distomatido, nome que não póde prevalecer por não encerrar ela nenhum genero de cujo nome este possa provir; deve ser adotada a designação de Hexamitidce, creada em 188082, por KENT; esta familia encerra dous generos de flajelados parasitos:

Generc Octomitus PROWAZEK, 1904. Trez flajelos anteriores de cada lado, dous flajelos caudaes, dous nucleos anteriores, Jous corpusculos basaes, tambem anteriores de onde saem os flajelos; os flajelos caudaes antes de se libertarem percorrem o corpo de uma a outra extremidade. HARTMANN e CHAGAS consideran os flajelados deste genero como formados pela fusão de dous Trichomastix; para admitir esta hipotese, é necessario que se admita o desaparecimento do axostilo, carateristico deste ultimo genero e que não tem similar em nerhum diplozoario parasito. Especie tipo: O. intestinalis PROWAZEK, 19.94.

Genero Giardia KUNSTLER, 1882. Dous nucleos anteriores, dous flajelos anteriores, dous lateraes, dous medianos, dous caudaes, um cromidio e uma ventosa anterior. Especie tipo; Giardia agilis KUNSTLER, 1882. A hipotese de HARTMANN e CHAGAS sobre a orijem de Octomitus ainda póde ser aplicada ao caso deste genero, havendo necessidade da restrição estabelecida no caso precedente e, mais ainda, de que se levem em conta as dificuldades decorrentes da presença de uma só ventosa e de um unico cromidio e da disposição complicada do aparelho nucleo-flajelar.

FAMILIA Oicomonadidae KENT, 1880-82,

SINONIMIA. Cercomonadidee KENT, $1880-82$, paj. 249, pro parte; Cercomonadina
BÜTSCHLI, 1884, paj. 812, pro parte; Otcomonadacece SENN, 1900, paj. 117; Oicomonadido KENT, 1880-82, paj. 250.

\section{Genero SPHAROMONAS (1) LIEBETANZ 1910.}

Sinonimia - Cercomonas DUJARDIN, 1841, in LJEBETANZ, 1910, paj. 35; Monas MULLER, 1786, in BRAUNE, 1914, paj. 122-123; Oicomonas KENT, 1880-82, in LIEBETANZ, 1910, paj. 33; Piromonas LIEBETANZ, 1910, рај. 37; BRAUNE, 1914, paj. 124-125; Spharomonas LIEBETANZ, 1910, paj. 25-26.

Historico - Reina grande confusão na sistematica dos flajelados do estomago dos ruminantes. Foram eles, até hoje, objeto de dous trabalho. $O$ primeiro de LIEBETANZ que, em 1910, distinguiu, na familia que denomina Cercomonadina, onze especies, distribuidas em quatro generos que parasitam ruminantes ; servem de criterio, para distinção generica, variações pequenas de fórma e, para caracteres especificos, diferenças de tamanho arbitrariamente consideradas. BRAUNE, em 1914, reduziu, a nosso ver com razão, todas essas fórmas a duas especies que, entretanto, dinda colóca em generos diferentes, e para isso se basea em diferença de fórma, constante mas pouco acentuada. Prociurando resolver a questão, preferimos admitir as duas especies aceiıas por BRAUNE, colocando, porém, ambas no genero Sphoromonas, o primeiro descrito por LIEBETANZ, visto como a nenhum genero anterior podem elas ser referidas. As especies admitidas por BRAUNE são Sphceromonas communis e Piromonas communis. Passando esta ultima para o primeiro genero, tem que mudar de designação especifica, por estar esta preocupada no genero Sphceromonas; propuzemos sua substituição pelo nome ae Sphoromonas liebetanzi, em homenajem ao autor que pela primeira vez a descreveu.

Diagnose - Corpo arredondado esferico ou piriforme, metabolico; nucleo cariosomico, esferico, ligado a um corpusculo basal por

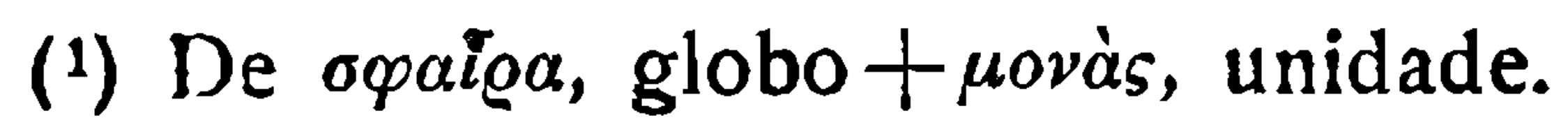


um rizoplasto; do corpusculo basal, colocado no polo anterior da celula, sae um longo flajelo encurvado para traz.

Morfolojia - Plasma distinto em endo e etoplasina; este é limitado externamente de maneira nitida por camada periplastica que permite ao parasito executar amplos movimentos metamorficos; o restante do etoplasma é hialino, desprovido de granulações e envolve completamente $o$ endoplasma. Este é alveolado; esta estrutura quasi sempre é mascarada pelo grande numero de granulações siderofilas vislumosas e esfericas de que está repleto o parasito.

Nucleo esferico, mais ou menos excentrico, ás vezes central; cariosoma volumoso, cercado da zona do suco nuclear vasia; não existe membrana nuclear que póde ser simulada por uma corôa de granulações siderofilas.

Corpusculo basal pequeno, anterior, submarjinal e ligado ao nucleo por meio de um rizoplasto. Desse corpusculo basal parte um flajelo recorrente, longo $\epsilon$ espesso que se vae afilando pouco a pouco para a extremidade livre.

Especies conhecidas:

$S$. communis LIEBETANZ.

S. liebetanzi, mihi, 1915.

Spharomonas communis LIEBETANZ, 1910.

Sinonimia. - Monas communis LIEBETANZ, in BRAUNE, 1914 pajs. 122-123; Oikomonas communis LIEBETANZ, 1910, paj. 33-34; O. minima LIFBETANZ, 1910, paj. 34; Sphoromonas communis LIEBE rANZ, 1914 ; S. maxima LIEBETANZ, 1910, pajs. 32-33; S. minima LIEBETANZ, pajs. 31-32.

Redescrição. - Corpo arredondado e muito metamorfico. Desse metamorfismo resultam aspetos variaveis nos preparados corados, frequentemente coincidindo com posições particulares do flajelo; dessas coincidencias resultou, provavelmente, a suposição erronea de multiplicidade de especies. Dimensões, habilualmente, $10 \mu \mathrm{de}$ diametrc, ás vezes baixa atê $7 \mu$ ou sóbe a $12 \mu$.
Plasma diferenciado em endo e etoplasma; este é nitidamente limitado para fóra e se continua para dentro com o endoplasma alveolado; o etoplasma é hialino, desprovido de granulações e muito flexivel, o que permite os movimentos metamorficos do protozoario; a estrutura alveolar do endoplasma é muitas vezes imperceptivel, por causa do grande numero de granulações siderofilas que a encobrem; essas granulaçōes são esfericas, volumosas e invisiveis a fresco.

Nucleo mais ou menos excentrico, esferico, cariosomico, com cerca de $2 \mu$ de diametro; zona do suco nuclear vasia, membrana nuclear ausente, mas ás vezes simulada por um colar de granulações siderofilas; cariosoma central, esferico, volumoso, que atinje ás vezes $1,5 \mu$ de diametro; zona do suco nuclear vasia e de coloração identica á do endoplasma com o qual se continua; não foi ainda visto centriolo.

Corpusculo basal sub-marjinal, esferico, pequeno e ligado ao nucleo por um rizoplasto; muitas vezes a zona do suco nuclear se extende na direção desse corpusculo.

Do granulo basal parte um flajelo espesso e muito longo, que tem cerca de $30 \mu$ de comprimento. Não estamos convencido da existencia do movimento que BRAUNE descreve para esse flajelo, não sendo, entretanto, improvavel que, como refere esse autor, muitas vezes o parasito execute movimento giratorio em torno do eixo antero= posterior. O flajelo é, então, dirijido para frente e parte de ponto um tanto lateral. Outro movimento giratorio, foi por nós observado não parecendc, porém, ser normal ao flajelado; é em torno de seu centro que o protista gira e o flajelo, a principio colocado em relação á celula como si fôra o prolongamento de um raio, de reto que era, vae-se encurvando á proporção qur aumentz a velocidade de rotação; em breve o protozoario e seu flajelo tomam o aspeto que LIEBETANZ representa na figura 1 da estampa 1, do trabalho citado.

O protozoario se reproduz por divisão binaria e por divisão multipla. 
Habitat. - Rumen de Bos talurus e céco de Cavia porcellus.

Sphceromonas liebetanzi, mihi, 1915.

Sinonimia. - Cercomonas rhizoidea communis LIEBETANZ, 1910, pajs. 35-36; C. r. maxima LIEBETANZ, 1910 , pajs. 3637 C. r. minima LIEBETANZ, 1910, paj. 36; Piromonas communis LIEBETANZ, 1910, pajs. 37-38; BRAUNE, 1914, pajs. 124-125, $P$. maxima LIEBETANZ, 1910 , pajs. $38-39$; P. minima LIEBETANZ, 1910, paj. 38.

Redescrição. - Corpo alongado, muitas vezes ovoide, outras vezes sub-cilindrico ou piriforme; metamorfismo menos acentuado que na especie precedente. Dimensões lrabituaes a $11 \mu$ de comprimento por 5 a 6 de largura; ás vezes o corpo atinje 15 a 16 $\mu$ de comprimento, por 5 a $6 \mu$ de largura.

Plasma diferenciado em endo e etoplasma; endoplasma cheio de granulações siderofilas menores e mais visiveis a fresco que na especie precedente; o etoplasma é nitidamente limitado para fóra e se continua insensivelmente para dentro com o endoplasma.

Nucleo, um pouco anteriormente colocado, cariosomico, muito refrinjente e, ao inverso do que acontece com a especie precedente, bem visivel. a fresco; cariosoma central, esferico e volumoso; zona do suco nuclear vasia; não exisłe membrana nuclear.

Flajelo espesso e muito longo, geralmente com mais de $30 \mu$; este flajelo parte de porção antero-lateral do corpo e se dirije em linha mais ou menos reta para traz. Durante o movimento, o flajelo é arrastado pelo protozoario que vae executando movimento rapido de rotação, em torno do eixo lonjitudinal do corpo.

O protozoario se multiplica por divisão binaria e por divisão multipla.

Habitat. - Rumen de Bos taurus.

Familia Callimastigidae, mihi, 1915.

\section{Genero (:ALLIMASTIX (1) WEISSEN- BERG, 1912. \\ Diagnose. - Corpo esferico, envolto por}

(1) De $x \dot{\alpha} \lambda v \zeta$, envolucro $+\mu \dot{\alpha} \sigma \tau \iota \zeta$, chicote; a etimolojia e a or tografia deste nome generico não estão de acordo uma com a outra; as regras de nomenclatura zoolojica nos impedem, porém, aqui qualquer correção. delgada capsula, numerosos flajelos anteriores mais ou menos paralelos, longos, partindo de corpusculos basaes, colocados lado a lado e ligados entre si por meio de rizoplastos.

Especies conhecidas:

C. cyclopis WEISSENBERO, 1912.

C. frontalis BRAUNE, 1914.

\section{Callimastix fronta:is BRAUNE, 1914.}

Redescrição. - Corpo geralmente esferico, raramente ovoide, não metamorfico. Dimensões médias, quando esferico, 9 a $10 \mu$; quando alongado, cerca de $11 \mu$ de comprimento, por 7 a $8 \mu$ de largura.

Plasima distinto em endo e etoplasma; este ultimo é externamente limitado por periplasta rijido e um tanto isolado do resto do corpo; o restante do etoplasma é pouco coravel e constitue camada continua, espessa, hialina e desprovida de granulações, envolvendo o endoplasma do qual é pouco nitidamente separada; o endoplasma tem estrutura alveolar pouco nitida, por causa do acumulo de granulações siderofilas volumosas e esfericas que ocupa todo o endoplasma.

Nucleo esferico, quasi sempīe central, volumoso e cariosomico; membrana nuclear nem sempre visivel, separada do cariosoma por zona do suco nuclear vasia; cariosoma volumoso com 1 a $1,5 \mu$ de diametro, intensamente corado; no centro do carisoma existe centriolo um tanto volumoso e cromófilo.

Da superficie da rejião anterior do protozoario, saem para frente, ao que parece, em grupos, numerosos flajelos finissimos e muitos longos, que atinjem 30 e mesmo $40 \mu$ de comprimento; eles têm sua orijem em corpusculos basaes pequenos, sub-marjinaes, ligados entre si por meio de finos rizoplastos; só cuidadosa diferenciação do preparado permite distinguir esses corpusculos das granulações siderofilas do endoplasma. Um desses corpusculos, pelo menos, póde estar ligado ao cariosoma do nucleo por meio de um rizoplasto.

Pela estrutura do plasma e pela presença de granulações siderofilas, este flajelado muito se assemelha ás $S$; iharomonas de que se distingue pelo numero, posição e dimensões 
dos flajelos e pela presença de envoltorio rijido.

Habitat. - Rumen de Bos taurus, de Capra hircus e de Ovis aries.

Familia Tetramitidae KENT, 1889-82.

Sinonimia. - Tetramitacece SENN, 1910, paj. 118; Tetramitida KENT, 1880-82, paj. 312; Tetramitına BÜTSCHLI, 1884, paj, 841 ; Trimastigidoe KENT, 1880-82, paj. 307, pro parte.

\section{Genero CHILOMASTIX (1) ALEXEIEFF, 1910.}

Sinonimia. - Cercomonas DIJJARDIN, 1841, paj. 287, in DAVAINE, 1884, pro parte; Cyathomastix PROWAZEK, 1914, paj. 162. Difcomus GÄBEL, 1914, paj. 18; Fanapepea PROWAZEK, 1911, paj. 97; Macrostoma ALEXEIEFF, 1909 (n. preoc. peixes); Monocercomonas GRASSI, 1881, paj. 11, pro parte; Tetramitus PERTY, 1852, paj. 170, pro parte; Trichomonas DONNÉ, 1837, in ROOS, 1893.

Diagnose.-Corpo mais ou menos piriforme, não metamorfico; o nucleo anterior tem, ás vezes, um cariosoma unido por um rizoplasto a corpusculo basal tambem anterior, donde partem: trez flajelos anteriores eguaes, o labio cromofilo do citostoma e uma membrana ondulante que percorre lonjitudinalmente esse citostoma.

Morfolojia. - Plasma distinto em endo e etoplasma. Este é constituido, quasi exclusivamente, por periplasta continuo, impedindo os movimentos metamorficos do protozoario; logo abaixo dele existe muitas vezes de'gada camada de plasma finamente granuloso. $O$ endoplasma é bastante alveolado, principalmente na porção media do corpo e contém poucas inclusões. Nunca existe axostilo, que, entretanto, é, ás vezes grosseiramente simulado por uma faixa de plasma correspondente ao eixo lonjitudinal do protozoario a qual, ás vezes, por estar situada entre fileiras de alveolos, se mostra constituida por uma linha mais intensamente corada ou por duas linhas

(1) De $\varkappa \varepsilon i l o s, ~ l a b i o+\mu \alpha \sigma \tau \iota \xi$, chicote. parzlelas mais coradas, que limitam um espaço mais claro.

O nucleo é anterior, um pouco lateral, marjinal, vesiculoso. Raramente existe cariosoma central que pode estar reunido ao corpusculo basal por meio de um rizoplasto; nesse caso a zona do suco nuclear é vasia ou, então apresenta esboço de reticulo de linina, constituido por filamentos acromaticos, que, partem do cariosoma, a modo de raios e vão terminar na membrana nuclear. Outras vezes, o que é mais frequente, não existe cariosoma: a cromatina do nucleo, neste caso, é representada por granulações lenticulares ou bacilares, aderentes á face interna da membrana nuclear. Esta membrana é espessa e muito cromófila.

O corpusculo basal, ora é unico, ora duplo; do corpusculo basal unico ou da granulação anterior, quando ele duplo, partem, para frente, os tre? flajelos eguaes entre si; do corpusculo basal unico, ou da granulação posterior do corpusculo duplo, parte o labio cromófilo e fortemente recurvado do citostoma, bem como uma membrana ondulante que percorre lonjitudinaln ente esse citnstoma.

O citostoma é bem visivel, tanto a fresco, como após coloração. A membrana ondulante, ao contrario, um tanto dificil de se perceber a fresco, é, geralmente, invisivel, após coloração; é muito provavel que tenha sido a pouco visibilidade da membrana o fato que levou GÄBEL a crear o genero Difcemus, o qual se distinguiria de Chilomastix, só pela ausencia da membrana ondulante.

\section{Sub-genero TETRACHILOMASTIX, mihi, 1915.}

O numero de flajelos do Chilomastix é passivel de oscilações. PROWAZEK descreveu um genero, Fanapepea que se distinguiria de Chilomastix só por ter, apenas, dois flajelos anteriores; entretanto, ele mesmo, ora representa o protozoario com dous, ora com trez flajelos anteriores, o que faz crer num erro de observação.

O mesmo não sucede com as pesquizas de MARTIN e ROBERTSON, que descreve- 
ram o Chilomastix gallinarum, como tendo quatro flajelos anteriores, e com as pesquizas de MACKINNON, que estabeleceu o genero Embadomonas, que se distingue de Chilomastix pela presença de um só flajelo anterior. No primeiro caso, a diterença não é bastante para formação de novo genero, mas nos parece justificar a creação de sub-genero, para o qual propuzemos o nome de Tetrachilomastix; desse modo procedendo, adoptámos o modo de ver daqueles que estabeleceram os sub-generos Tetratrichomonas e Tetratrichomastix. Quanto ao genero Embadomonas, além da diferença numerica de flajelos mais acentuada, existem tambem outras pequenas diferenças morfolojicas que justificam a separação desses flajelados em genero distinto.

A especie unica deste sub-genero será Chilomastix (Tetrachilomastix) gallinarum MARTIN et ROBERTSON, 1911.

Especies descritas:
C. bittencourti, mihi, 1915.
C. bocis BRUMPT, 1912.
C. capro, mihi, 1915.
C. caulleryi (ALEXEIEFF, 1909).
C. cuniculi, mihi, 1915.
C. gallinarum MARTIN et ROBERTSON, $191 \mathrm{k}$.

C. intestinalis KUCZYNSKI, 1914.

C. mesnili (WENYON, 1910).

C. motellae ALEXEIEFF, 1912.

\section{Chilomastix bittencourti, mihi, 1915.}

Redescrição. - Corpo alongado, ovoide, de contornos geralmente menos regulares que nas outras especies do genero; dimensões : lonjitudinalmente, 13 a $16 \mu$, excecionalmente até $11 \mu$; transversalmente $9-11 \mu$, excecionalmente até $7 \mu$. E' a maior especie do genero que parasita mamiferos.

Extremidade anterior arredondada e romba; extremidade posterior afilada em ponta ou, ás vezes, tambem arredondada, sempre, porém, mais delgada que a anterior. $\mathrm{Na}$ extremidade anterior existe citostoma com labio fortemente cromofilo, de 4 a $5 \mu$ de comprimento, por 2 a $3 \mu$ de largura. Frequen- temente parece este, nos preparados corados, se enrolar em torno do nucleo.

De um corpusculo basal, colocado adeante e a um dos lados do nucleo, sae para traz uma membrana ondulante bem visivel, a iresco, percorrendo o citostoma, cujo labio tem a mesma orijem. Do mesmo corpusculo basal, ou de outro colocado a seu lado, saem, para deante, trez delgados flajelos, mais ou menos do tamanho do corpo.

Protoplasma distinto em endo e etoplas. ma. O etoplasma é constituido sómente pelo periplasta, cuja camada continua e finamente granulosa apresenta rijidez bastante para impedir movimentos metamorficos do corpo. O endoplasma é alveolado e nas paredes dos alveolos existem finas granulações. A fresco, parece que este flajelado se apresenta com aspeto mais granuleso que o Chilo. mastix intestinalis.

Nucleo anteriormente colocado, ás vezes um tanto lateral, com 3 a $4 \mu$ de diametro; membrana nuclear espessa e nela se prendem massas cromaticas, de fórma mais ou menos lenticular, em numero de uma a duas, sendo então, uma delas, por vezes, maior que a outra. Zona do suco nuclear vasia. Raramente aparece cariosoma central volumoso. Não coexiste essa formação, de modo frequente, com as massas cromaticas da periferia.

Habitat. - Céco de Mus (Epimys) norwegicus.

Nota. - A designação especifica foi dada em homenajem ao Prof. Dr. NASCIMENTO BITTENCOURT, catedratico de Historia Natural Medica e iniciador do ensino oficial da Parasitolojia na Faculdade de Medicina do Rio de Janeiro.

\section{Chilomastix capro, mihi, 1915.}

Redescrifão. - Corpo alongado, mais ou menos piriforme, largo anteriormente $e$ se estreitando, ás vezes bruscamente, a partir dos dous terços posteriores. Corpo não melamorfico, raramente deformavel, com cerca de 9 a $12 \mu$ de comprimento por 5 a $6 \mu$ de largura.

A extremidade anterior arreaiondada, apre- 
senta uma depressão lateral, quasi paralela á borda lateral do corpo. Extremidade posterior afilada em cauda, geralmente curta; raramente a extremidade posterior é arredondada.

Protoplasma distinto em endo e etoplasIna; este constituido só pelo periplasta rijido, cuja existencia impede os movimentos metamorficos do protista; endoplasma alveolado com poucas inclusões; nos dous terços anteriores os alveolos são grosseiros e volumosos e se tornam delicados e pequenos no terço posterior.

$\mathrm{Na}$ extremidade anterior existe o citostoma bem visivel, a fresco, com labio cromofilo nitido nos preparados corados; citostoma geralmente pouco encurvado, com cerca de 3 a $4 \mu$ de comprimento por 1 a $2 \mu$ de largura. E' percorrido por membrana ondulante cuja orla não cromofila é constituida por flajelo aderente que parte de um corpusculo basal sub-marjinal colocado anteriormente em relação ao nucleo e donde sae tambem o labio do citostoma.

Este nucleo é anterior, sub-marjinal, esferico, pobre em cromatina; ás vezes parece sub-jasente ao citostoma, ou viceversa; frequentemente é um tanto lateral ; cariosoma muitas vezes visivel, ligado ao corpusculo basal por um rizoplasto; cromatina nuclear grupada sob a fórma de massas em bastonete recurvado, aderentes á face interna da membrana nuclear e, geralmente, em numerc superior a duas; frequentes vezes essas granulações estão reunidas ao cariosoma por filamentos de linina que formam raios que partem do centro para a periferia do nucleo.

Flajelos anteriores trez, de egual comprimento, partindo do mesmo corpusculo basal unico que dá orijem ao labio cromófilo do citostoma e á orla não cromófila da membrana ondulante.

Habitat. - Rumen de Capra hircus.

Nota. - BRAUNE descreve na pansá dos ruminantes dous flajelados sobre cuja posição sistematica fornece dados positivamente erroneos. Um deles é um Trichomastix que não tivemos ocasião de observar e que o autor coloca no genero Trichominas.
O outro, é um flajelado com trez flajelos anteriores, sem flajelo recorrente, sem membrana ondulante e sem citostoma; não o pudemos identificar ao nosso Chilomastix, do qual difere por muitos caracteres; BRAUNE o julga Trichomastix.

\section{Chilomastix cuniculi, mihi, 1915.}

Redescrição. - Flajelado piriforme, geralmente estreito e alongado, raramente largo e mais arredondado e curto. Dimensões medias: 7 a $9 \mu$ de comprimento, por 4 a $7 \mu$ de largura.

Extremidade anterior arredondada e larga junto á qual, ao lado do citostoma, existe uma depressão mais ou menos acentuada. Extremidade posterior afilada em longa cauda que pode, por vezes, atinjir tamanho egual á metade do comprimento do corpo; raramente, a cauda é curta ou não existe, sendo, então, a extremidade posterior do corpo arredondada.

Plasma, em geral, delicadamente alveolado. Periplasta rijido que impede os movimentos metamorficos do corpo; limite externo nitido, constituindo o periplasta só, todo o etoplasma.

$\mathrm{Na}$ extremidade antetior existe citostoma com labio cromofilo; o citostoma tem 5 a 3 $\mu$ de comprimento, por 1 a $2 \mu$ de largura, sendo frequentemente encurvado.

Nucleo esferico, colocado na extremidade anterior do parasito, ás vezes um tanto lateral, getalmente sub-marjinal, com $1,5 \mu$ de diametro. Membrana nuclear espessa; o cariosoma, quando existe, é muito pequeno; neste caso a zona do suco nuclear frequentemente apresenta filamentos acromaticos, geralmente um numero de trez, ligando o pequeno cariosoma á membrana nuclear. Nesta existem frequentemente, aderentes a sua face interna, trez a quatro granulações de cromatina, irregulares e alongadas.

Flajelos menores que o corpo, em numero de trez anteriores e eguaes em comprimento e espessura, purtindo de corpusculo basal colocado anteriormente, em relação ao nucleo. Do mesmo corpusculo basal, parte para traz o flajelo aderente á membrana ondulante. 
Esta é bem visivel a fresco, e percorre o citostoma. culus.

Habitat. - Céco de Oryctolagus cuni-

Nota. - Nenhuma referencia encontrámos na bibliografia, a flajelados do céco do coelho; o parasito que descrevemos não é o unico que aí se encontra, não sendo tambem rara sua ocorrencia.

Chilomastix intestinalis KUCZYNSKI, 1914.

Sinonimia. - Trichomonas cavice DAVAl$\mathrm{NE}, 1854$, pro parte, auctorum.

Historico. - Este protozoario foi descoberto, representado e classificado em 1914 por KUCZYNSKI, que o cita em seu trabalho sobre os Trichomonas; a primeira descrição desta especie foi por nós publicada em 1915 e é a que abaixo transcrevemos.

Descrição. - Corpo alongado, piriforme, de 13 a $16 \mu$ de comprimento, por 7 a $9 \mu$ de largura; extremidade anterior muito mais larga que a posterior, na qual, ás vezes, existe expansão caudal. Corpo não metabolico, ás vezes, entretanto, deformado nos preparados, nunca porém, tanto quanto os individuos do genero Trichomonas.

$\mathrm{Na}$ extremidade anterior existe citostoma de labio facilmente coravel, com 4 a $6 \mu$ de comprimento, largamente aberto para diante depois estreitado, vindo se alargar em saco na porção posterior em que atinje $3 \mu$ de largura. Dentro dele existe membrana ondulante, que parte do corpusculo basal; essa membrana é visivel a fresco e muito dificilmente coravel. Não ha flajelo livre posterior.

Do mesmo corpusculo basal anterior, partem, para diante e um tanto para um lado, trez flajelos finos e de egual comprimento.

Protoplasma mais ou menos grosseiramente alveolado nos $3 / 5$ medios do corpo; ha diferenciação nitida entre endo e etoplasma; os limites externos do corpo são claramente assinalados pelo periplasta que, só ele, constitue todo o etoplasma e cuja consistencia impede os movimentos metamorficos do protozoario; endoplasma alveolado contendo geralmente poucas inclusões.
Nucleo anterior, colocado junto ao citostoma ; membrana nuclear espessa, cariosoma, em geral, ausente, cromatina quasi sempre disposta em massas aderentes á face interna da membrana nuclear; ás vezes, essas massas são duas e estão colocadas em polos opostos do nucleo.

Habitat. - Céco de Cavia porcellus.

Chilomastix mesnili (WENYON, 1910).

Sinonimia. - Cercomonas hominis DAVAINE, 1854, pro parte. Cyathomostix hominis PROWAZEK, 1914, paj. 162. Diformus tunensis GÄBEL, 1914, paj. 18; Fanapepea intestinalis PROWAZEK, 1911, paj. 97; Macrostoma mesnili WENYON, $1910 \mathrm{~b}$; Tetramitus mesnili, (WENYON, 1910) auctorum.

Historico. - Este parasito foi visto e confundido com outros por quasi todos os autores que estudaram flajelados intestinaes do homem; GRASSI, EPSTEIN e MARCHAND o representam e descrevem, ora no genero Monocercorronas, ora no genero Cercomonas; WENYON o estuda corretamente e o descreve no genero Macrostoma, depois mudada por ALEXEIEFF esta denominação para a de Chilomastix; PROWAZEK, em 1911, descreve um novo genero Fanaperea, com a especie $F$. intestinalis que se distinguiria de Chilomastix por ter apenas dous flajelos; o mesmo autor, entretanto, a representa, ora com dous, ora com trez flajelos, o que nos faz colocar esse genero na sinonimia; GÄBEL descreve Difcemus tunensis que difere do parasito em questão pela ausencia de membrana ondulante cuja dificil visibilidade, faz crer em facil erro de observação; RODENWALDT confunde o Chilomastix com - Trichomonas do homem e representa esquematicamente aquele com um axostilo deste, o que, certamente, não existia; para esta ultima fórma, PROWAZEK creou, em 1914, o Cyathomastix hominis, dificilmente aceitavel.

Encontrado pela primeira vez no Brazil, pelos Drs. ARISTIDES MARQUES da CUNHA e MAGARINOS TORRES, $\epsilon \mathrm{m}$ casos de disenteria verificados no ano de $1914 \mathrm{em}$ Lassance, Estado Minas Geraes, foi por nós observado em fezes disentericas provenientes 
dos serviços de pediatria do Hospital da Santa Casa da Misericordia do Rio de Janeiro.

Redescrif̧ão. - Flajelado mais ou menos piriforme raramente um tanto arredondado, com 10 a $15 \mu$ de comprimento, raras vezes menos, e cerca de 4 a $7 \mu$ de largura.

Extremidade anterior larga e arredondada; a extremidade posterior se afila em cauda, a partir do porito de união do terço médio com o terço posterior do parasito; ás vezes, a cauda é longa e termina ponteaguda; mais raramente é ela arredondada.

Plasma distinto em endo e etoplasma; aquele é alveolado e contém poucas inclusões; este é em geral constituido quasi exclusivamente pelo periplasta.

$\mathrm{Na}$ extremidade anterior está o nucleo esferico, sub-mariinal, com 1 a $3 \mu$ de diametro, raramente com cariosoma central; zona do suco nuclear em geral vasia; uma a duas granulações de cromatina presas á membrana nuclear.

Na mesma extremidade está o citostoma com labio cromófilo e com 3 a $4 \mu$ de comprtmento por 1 a $2 \mu$ de largura .O labio do citostoma parte de corpusculo basal unico, anterior ao nucleo, pequeno, que tambem é o ponto de orijem da membrana ondulante. Esta percorre o citostoma lonjitudinalmente, é bém visivel a fresco e pouco coravel.

Flajelos anteriores em numero de trez, com igual comprimento e partindo do mesmo corpusculo donde saem a membrana ondulante e o labio do citostoma.

Habitat. - Intestino de Homo sapiens.

Genero Trichomonas (1) DONNÉ, 1837.

Sinonimia. - Bodo EHRENBERG, 1838 in KENT, 1880-82; Cercomonas DUJARDIN, 1841, paj. 287, in DAVAINE, 1834; Cimcenomonas GRASSI, 1881, paj. 11, pro parte; Entamaba LEIDY, 1879, in CASTELLANI, 1905; Exechlyga STOKES, 1884; Löschia CHATTON et LALUNG-BONNAIRE, 1912 ; Monocercomonas GRASSI, 1881, paj. 11, pro dade. parte; Schedaocercomonas ORASSI, 1879, pro parte.

Diagnose. - Corpo ovoide, muito metabolico; dum corpusculo basal anterior partem trez flajelos livres, para deante, e para traz, um flajelo aderente a uma membrana ondulante que percorre extensão variavel da superficie do corpo; nucleo antęrior, raramente cariosomico; axostilo saliente para o exterior, ás vezes se continuando com o citostoma; este é juxta-nuclear e não tem labio cromófilo.

Especies conhecidas:

$T$. ardin delteili (DERIEU et RAYNAUD, 1914.).

T. augusta ALEXEIEFF, 1911.

T. batrachorum PERTY, 1852.

T. brumpti ALEXEJEFF, 1912.

T. cavioe DAVAINE, 1875.

T. columbarum PROW/AZEK e ARAGAO, 1909.

T. eberthi MARTIN et ROBERTSON, 1911.

T. gallinarum MARTIN et ROBERTSON, 1911.

T. granulosa ALEXEIEFF, 1914.

T. hominis DAVAINE, 1854.

T. Lacerta PROWAZEK. 1904.

T. mabuia DOBELL, 1910.

T. muris GALLI-VALERIO, 1907.

$T$. parva ALEXEIEFF, 1911.

T. prowazeki ALEXEIEFF, 1909.

T. tatusi, mihi, 1915.

T. tritonis ALEXEIEFF, 1911.

T. vaginalis DONNÉ, 1837.

Morfolojia. - Protoplasma mais ou menos nitidamente alveolado; periplasta delgadissimo, constituindo, só ele, todo o etoplasma, permitindo os movinentos metamorficos intensos do protozoario. No polo anterior o plasma é mais intensamente corado pelo acumulo maior, que ai se verifica, de granulações siderofilas e diversos derivados nucleares. Num dos bordos se prende a membrana ondulante, delgadissima lamina etoplasmatica, em relação direta com o aparelho flajelar.

No interior do plasma existe frequentemente um vacuolo, ás vezes vasio e incolor, 
outras vezes ocupado por um corpusculo que se córa em azul mais ou menos intenso pelo processo de HEIDENHAIN; ás vezes, esse corpusculo é esferico, ovoide ou mais ou menos alongado; outras vezes, ele tem a fórma de hexagono regular de angulos arredondados; esta formação foi por nós encontrada em Trichomonas cavia e T. muris, e foi vista algumas vezes fóra do corpo de qualquer protozoario, livre no material examinado; nenhuma referencia encontrámos na bibliografia, sobre os corpusculos em questão, julgamol-os entretanto, de natureza cromatoide; facil é diferencial-os da cromatina pelo colorabilidade analoga mas sempre menor, que apresentam; esta formação persiste nos protozoarios em divisão.

Granulações siderofilas em numero e de volume variaveis existem, ocupando, ás vezes, posições carateristicas das diferentes especies de Trichomonas. Atravessando o corpo de um a outro lado, existe um bastonete espesso que, ás vezes, parece se continuar por uma de suas extremidades com um citostoma em fórma de fenda colocado anteriormente ao lado do nucleo; o outro extremo ponteagudo e frequentemente cortado en bisel faz saliencia para o exterior. Os limites entre o plasma e o axostilo são sempre nitidos, talvez pela maior refrinjencia deste, talvez, o que parece ocorrer em algumas fórmas, pela existencia de uma linha limitante mais intensamente corada que aquelas duas formações; o interior do axostilo é sempre incolor, podendo, entretanto, conter granulações siderofilas, $o$ que se torna carater importante. No ponto em que o axostilo se desprende do plasma para fazer saliencia, para o exterior, em varias especies, se nota a presença de duas granulações cromaticas baciliformes nos dous bordos do axostilo. A orijem do axostilo é extremamente controvertida; para alguns, DOBELL, por exemplo, é derivado da centrodesmose dos corpusculos basaes, para outros deriva do nucleo e, para outros, emfim, MARTIN e ROBERTSON, por exemplo, nada tem que ver com o aparelho nuclear; a confusão é muito aumentada por julgarem quasi todos os autores como homologas as formações esqueleticas dos Trichomonas e Trichomastix e as das Cercomonas (HARTMANN e CHAGAS), Hexamitus, etc.; a nosso ver se trata de duas formações completamente distintas; sobre a natureza e orijem da primeira. nada podemos de seguro adiantar; quanto á segunda, esta é indubitarelmente derivada do nucleo, e suas relações morfolojicas e funcionaes com o aparelho flajelar são indiscutiveis. Voltaremos ao assunto quando estudarmos os diplozoarios.

O citostoma, em fórma de fenda, é juxta-nuclear e colocado na base dos flajelos; ele frequentemente se continua de modo completo com o axostilo.

Nucleo anterior, raramente cariosomico e com centriolo; mais frequentemente $o$ nucleo é constituido por um grupo de granulaçíes cromaticas esparsas no pólo anterior do parasito. Zona do suco nuclear é vasia e coexiste com o cariosoma o qual é ás vezes muito volumoso. Adeante do nucleo, junto á borda do protista, existe corpusculo basal unico ou duplo; desse corpusculo on de sua granulação anterior partem os flajelos anteriores em numero de trez para as fórmas tipicas do genero, em numero de quatro, no sub-genero Tetratrichomonas, e de cinco no sub-genero Fentatrichomonas; do mesmo corpusculo, ou de sua granulação posterior, parte o flajelo recorrente que adere em toda ou quasi toda sua extensão á membrana ondulante; esta assenta sobre uma barra cromófila, a costa, que parte da mesma granulação donde sae o flajelo aderente, que se denomina orla da membrana ; 1) flajelo aderente, ás vezes, se continua além da membrana ondulante, e essa porção ecedente se denomina flajelo livre; a costa é flexivel, reta ou encurvada, conforme o corpo do protozoario está estirado ou arredondado; ás vezes a costa é dupla, o que pode suceder quando ocorre duplicidade dos corpusculos basaes.

Como anexo do aparelho nuclear, ás vezes parte do corpusculo basal, um bastonete cromatico, morfolojicamente variavel, 
que recebeu de JANICKI o nome de aparelho para-basal.

Trichomonas cavice DAVAINE, 1875.

Sinonimia. - Cercomonas ovalis PERRONCITO, 1888; C. pisiformis PERRONCITO, 1888. Trichomonas intestinalis LEUCKART, 1879, auctorum.

Descrição. - Corpo de fórma variavel e muito metabolico; quando não deformado, mais ou menos ovoide, de 10 a $15 \mu$ de comprimento.

$\mathrm{Na}$ parte anterior do corpo existe citostoma sem labio cromófilo, em fórma de fenda, mais ou menos triangular e encurvada, pequena e que se continua para traz com o axostilo, pouco coravel e saliente para a parte posterior do corpo; axostilo tem a extremidade posterior cortada em bisel, ponta afilada e longa e mede cerca de $10 \mu$ de comprimento por 0,5 a $1,0 \mu$ de largura. Frequente é a presença do vacuolo e da granula̧ão cromatoide que já descrevemos.

$\mathrm{Na}$ extremidade anterior existe um corpusculo basal donde parte $m$, para frente trez finos flajelos mais ou menos longos que o corpo e, para traz, o flajelo recorrente muito espesso e preso, em certa extensão, á membrana ondulante; o flajelo recorrente é longo e atinje ás vezes $30 \mu$ de comprimento. Membrana ondulante com cinco a oito em geral seis, ondulações muito acentuadas, percorrendo pouco mais de metade da circumferencia do corpo. Costa espessa e flexivel, muito refrinjente e bem visivel a fresco, fortemente encurvada nas fórmas arredondadas e quasi reta nas fórmas alongadas, ás vezes, em sua extremidade posterior mais ou menos ondulada; a costa é acompanhada por uma fileira de granulações cromófilas.

Protoplasma difereliciado em etoplasma, constituido sómente pela delgadissima camada periplastica e endoplasma mais ou menos delicadamente alveolado.

Nucleo colocado anteriormente entre o citostoma e a costa da membrana ondulante; ás vezes, com cariosoma volumoso e, ás vezes, centriolo e aspetos assimilaveis á evolução ciclica do cariosoma.

Fórmas de divisão com dous corpusculos basaes e duas costas reunidas pela parte anterior e, ás vezes, tambem pela parte posterior, estas fórmas são, em geral, muito maiores e atinjem 18 a $24 \mu$ de comprimento por 14 a $17 \mu$ de largura; ha casos, porém, em que fórmas dessas dimensões não têm costa e corpusculo basal duplos.

Habitat. - Céco de Cavia porcellus e C. aperea.

Trichomonas hominis (DAVAINE, 1854).

Sinonimia. - Bodo hominis DAVAINE in KENT, 1880-82, paj. 256; Cercomonas hominis DAVAINE, 1854, pro parte; Cimonomonas hominis GRASSI, 1882, paj. 11; Monocercomonas hominis GRASSI, 1881, pajs. 12-23, pro parte; Trichomonas intestinalis LEUCKART, 1879; Trichomonas buccalis, auctorum; T. dysenterioe BIL,LET, 1907; T. pulmonalis SCHIMIDT, 1895.

Redescrição. - Flajelado muitissimo metamorfico, em geral piriforme ou arredondado, com 5 a $10 \mu$ de comprimento por 2 a $3 \mu$ de largura ou mais, quando arredondado.

Plasma mal distinto em endo e etoplasma, representado este apenas pela delgada camada etoplasmatica, cuja flexibilidade permite os intensos movimentos metamorficos do protozoario; endoplasma delicadamente alveolado e contendo poucas inclusões.

Citostoma em fó:ma de ienda larga, parecendo muitas vezes se continuar diretamente com o axostilo; este é muito nitido, calibroso, longo, ponte-agudo e saliente em sua extremidade posterior, não sendo acompanhado por granulações siderofilas que tambem não existem em seu interior.

Ao lado do citostoma, está o nucleo esferico ou ovoide, muitas vezes cariosomico, outras vezes constituido por granulações cromaticas esparsas; o nucleo atinje cerca de $1,5 \mu$ de diametro e, quando existe cariosoma, tem zona do suco nuclear vasia.

Dum corpusculo basal anterior, ligado ao cariosoma do nucleo por um rizoplasto, 
saem para frente trey flajelos geralmente maiores que o corpo e não raro reunidos em feixes junto a seu ponto de emerjencia. Do mesmo corpusculo basal, parte, para traz, o flajelo aderente presc ao corpo por uma membrana ondulante que tem cerca de cinco ondulações pouco profundas; este flajelo recorrente é maior e mais espesso que os anteriores e, ás vezes, constitue em sua porção terminal flajelo livre. A membrana ondulante percorre lonjitudinalmente cerca de metade da circumferencia do corpo.

Trichomonas muris GALLI-VALERIO, 1907.

Sinonimia. - Trichomonas intestinalis LEUCKART, 1879, auctorum.

Historico. - Foi visto em 1885 por KUNSTLER que não verificou a especie a que pertencia o parasito, nem lhe estudou a morfolojia.

Descrição. - Corpo de fórma quasi sempre ovoide, ou mais ou menos alongado; em geral de aspeto mais regular e de fórma mais alongada que o Trichomonas cavia; dimensões, Ionjitudinalmente, 13 a $18 \mu$, transversalmente 6 a $9 \mu$.

$\mathrm{Na}$ extremidade anterior, está o citostoma aberto anteriormente e que se continua quasi sempre posteriormente com o axostilo vasio de granulaçōes; este é geralmente encurvado, estando a convexidade da curva voltada para a membrana ondulante; o axostilo é muito visivel, saliente para o exterior, com a ponta biselada, provido de duas granulações bacilares no ponto em que se desprende do plasma para fazer saliencia para o exterior; este axostilo atinje $12 \mu$ de comprimento, por $0,5 \mu$ de largura.

No polo anterior do corpo está um corpusculo basal, sub-marjinal, donde partem trez flajelos anteriores relativamente curtos; para traz sae o flajelo recorrente espesso, servindo de orla á membrana ondulante e se tornando depois flajelo livre em pequena extensão; a membrana ondulante tem cerca de oito ondulações muito profundas e percorre bastante exatamente metade da circumferencia do corpo do flajelado; ela se apoia sobre uma costa espessa, ás vezes dupla, partindo do mesmo corpusculo que a orla.

Protoplasma alveolar mal diferenciado em endo e etoplasma; granulações siderofilas esparsas no plasma, havendo constantemente uma fileird delas que acompanha a costa da membrana ondulante, do lado do nucleo; outras granulações siderofilas formam uma figura conica $e$ encurvada na parte anterior do parasito, indo da rejião peri-nuclear até a parte media ou posterior da celula. Outras granulaçōes siderofilas formam curta fileira de cada lado da porção anterior do axostilo.

O citostoma anterior tem a fćrma de fenda larga que parece continuar com o axostilo.

Entre o citostoma e a costa está o nucleo, ás vezes com um grande cariosoma e a zona do suco nuclear vasia, outras vezes não individualizado e constituido por granulações cromaticas mais ou menos esparsas; estas granulações, ás vezes, se apresentam em camadas circulares concentricas ás vezes em redor de um pequeno granulo; dá esse aspeto impressão dos fenomenos de evolução ciclica do ca. riosoma.

Habitat. - Céco de Mus norwegicus e sua fórma albina, de Mus rattus e de Mus musculus e sua fórma albina.

Trichomonas tatusi, mihi, 1915.

Descrição. - Corpo ovoide ou mais ou menos arredondado, com cerca $10 \mu$ de comprimento por $6 \mu$ de largura, muito r.etamorfico.

Plasma delicadamente alveolar, mal distinto em etoplasma, constituido apenas pela delgadissima camada periplastica e em endoplasnia que encerra poucas inclusões.

Citostoma anterior, juxta-nuclear, em fórma de estreita fenda. Axostilo saliente para o exterior e visivel a fresco.

$\mathrm{Na}$ rejião correspondente'ao nucleo, o qual não vimos individualizado, se encontram granulaçōes cromaticas mais ou menos irregulares situadas entre o citostoma e a membrana ondulante.

De um corpusculo basal anterior, partem 
para frente trez flajelos livres, eguaes, del. gadissimos e maiores que o corpo. Do mesmo corpusculo basal parte o flajelo recorrente pouco espesso, preso ao corpo por uma delgadissima membrana ondulante, cujas ondulações são largas, pouco profundas e geralmente em numero de duas ou trez; a membrana ondulante percorre cerca de metade da circumferencia do corpo e, quando termina, sua orla vae em geral constituir um longo flajelo livre. Do mesmo corpusculo basal, parte a costa sobre a qual repousa a membrana ondulante; é uma barra cromatica pouco espessa que acompanha a superficie do corpo em toda a extensão da membrana ondulante.

Assistimos á divisão transversal no protozoario vivo.

Habitat. - Parte terminal do intestino de Tatus novemcinctus.

Trichomonas vaginalis DONNÉ, 1837. 1858.

Sinonimia. - $T$. irregularis SALISBURY,

Redescrição. - Flajelado muito maior e menos metamorfico que o Trichomonas hominis com cerca de 12 a $16 \mu$ de diametro, geralmente arredondado ou ovoide.

Endoplasma alveolado, contendo poucas inclusões, limitado externamente pela delgada camada periplastica que, só ela, constitue todo o etoplasma e cuja delgadêza permite os movimentos mntamorficos do protozoario. Axostilo saliente e pouco visivel. Citostoma anterior, em fórma de fenda.

Nucleo anterior e, adeante dele, o corpusculo basal simples ou duplo donde partem para frente trez flajelos livres, delgados, eguaes e mais ou menos do tamanho do corpo: para traz, sae do mesmo corpusculo basal o flajelo aderente espesso, preso ao corpo por uma membrana ondulante; esta percorre cerca de um terço da superficie do corpo, é muito estreita e tem cerca de seis ondulações bem nitidas e acentuadas.

Habitat. - E' parasito inofensivo da vajina da mulher, da qual póde emigrar para o aparelho urinario e penetrar mesmo na bexiga onde, segundo alguns, póde concorrer, para a persistencia de certas cistites. Tivemos ocasião de ver flajelados desse genero na urina dum homem internado, ha muito, no Hospital da Misericordia, do Rio de Janeiro.

\section{Genero Trichomastix (1) BLOCHMANN 1884.}

Sinonimia. - Cercomonas DUJARDIN, 1841 in PERRONCITO, 1888, pajs. 220221 ; Heteromita GRASSI, 1881, paj. 12, Monas (?) MULLER, 1786, in DAVAINE, 1875; Trichomonas DONNÉ, 1837, in DOFLEIN.

Especies descritas :

Trichomastix cavice (GRASSI, 1882).

T. lacertae (BÜTSCHLI, 1884).

T. mabuice DOBELL, 1910.

T. motella ALEXEIEFF, 1910.

T. orthopteroi um (PARISI, 1910).

T. salpce ALEXIJIEFF, 1914.

T. serpentis DOBELL, 1907.

T. trichopterce MACKINNON, 1910.

Trichomastix cavice (GRASSI, 1881)

Sinonimia. - Cercomonas globosus PERRONCITO, 1888; Heteromita cavia GRASSI, 1882, paj. 35; Monas caviae (?) DAVAINE, 1875.

Redescrição. - Corpo geralmente piriforme, mais raramente arredondado; extremidade anterior arredondada, a extremidade posterior gradualmente se afilando até terminar em ponta que corresponde, quasi sempre, á parte terminal do axostilo. As dimensões médias ocilam entre 7 a $9 \mu$ de comprimento, por 4 a $7 \mu$ de largura.

$\mathrm{O}$ axostilo tem o aspeto de um tubo completamente vasio, mais ou menos retilineo de que uma extremidade faz saliencia para o exterior ao passo que a outra se perde na massa plasmatica, parecendo, ás vezes, se continuar com o citostomá. Este aparece, como fenda mais ou menos conica e encurvada, num ponto antero-lateral da celula,

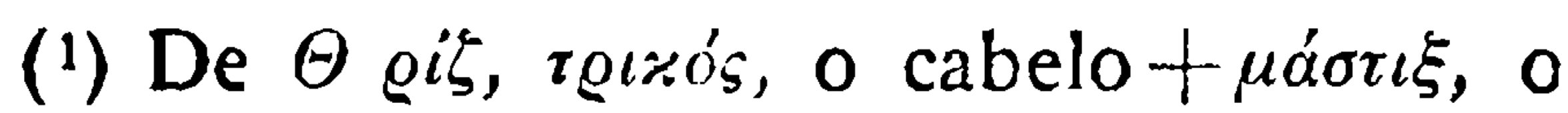
chicote. 
junto á base dos flajelos; não existe labio cromófilo.

O plasma não é nitidamente distinto em endo e etoplasma; este é representado, apenas, por delgadissima camada periplastica, cuja flexibilidade permite ao protozoario realisar intensos movimentos metamorficos; o endoplasma é delicadamente alveolado e contém poucas inclusões; não existem granulaçôes siderofilas.

O nucleo é cariosomico, anterior, submarjinal, esferico ou, ás vezes, de fórma irregular; o cariosoma tem volume variavel e pode atinjir 2 a $3 \mu$ de diametro; zona do suco nuclear vasia; parece existir membrana nuclear.

Um corpusculo basal, ligado ao nucleo por um rizoplasto dá orijem a trez flajelos anteriores, menores que o corpo do flajelado e a um flajelo recorrente livre maior do que ele.

Habitat. - Céco de Cavia aperea, C. porcellus e Dasyprocta aguti.

\section{Genero Enteromonas ( $\left.{ }^{1}\right)$ mihi, 1915.}

Diagnose. - Protomonadinas com um flajelo maicr recorrente e livre e dous menores anteriores, eguaes; corpo globoso sem axostilo, sem citostoma e sem mem. brana ondulante.

Enteromonas hominis, mihi, 1915.

Redescriçâo. - Flajelado de corpo quasi sempre regularmente esferico, ás vezes com a extremidade posierior afilada em cauda muito curta. Dimensões médias 5 a $6 \mu$ de diametro.

Periplasta delgado, porém, suficientemente rijido para impedir movimentos metamorficos do prolozoario e, só ele, constitue todo o etoplasma. Endoplasma alveolado, contendo frequentemente inclusões muitas vezes constituidas por bacterios. A disposição dos alveolos, não raro, é regular, sendo que, então, nos preparados córados, um deles aparece proximo ao centro do parasito,

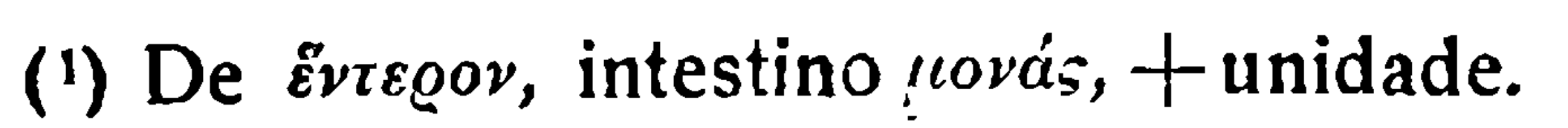

emquanto os outros formam uma corôa em torno dele. Não existem axostilo, nem citostoma.

Nucleo anterior, esferico, sub-marjinal ou, ás vezes, central, com $1 \mu$ de diametro. $\mathrm{O}$ nucleo é de tipo protocarion, isto é vesiculoss, com cariosoma e zona do suco nuclear vasia, sem membrana nuclear. Cariosoma central, volumoso e esferico, raramente irregular ou pequeno. Zona do suco nuclear vasia e muito estreita. Não foi visto centriolo.

Flajelos em numero de trez, sendo um recorrente e maior que o corpo e os outros anteriores menores que ele. Os flajelos prrtem de corpusculo basal unico, muito pequeno sub-marjinal, colocado anteriormente em relação ao nucleo, ao qual está unido por meio de rizoplasto.

O flajelado se reproduz por divisão lonjitudinal; nas primeiras fazes desta, aparecem duas placas cromaticas, em que parece haver distinção de cromosomas; dous corpusculos basaes dão, nessas fórmas, orijem a dous grupos di: flajelos; não raro, entre as placas cromaticas se observa centrodesmose que póde ser bastante espessa.

Raramente são encontradas fórmas grandes com numerosos flajelos irregularmente dispostos; talvez possam essas fórmas ser interpretadas de acordo com a opinião de HARTMANN e CHAGAS, sobre dissociação dejenerativa das fibrilas constituintes do filamento axial de cada flajelo.

Habitat. - Intestino de Homo sapiens; o flajelado foi observado em fezes emitidas, menos de cinco minutos antes de exame, por uma doente do Hospital Nacional de Aiienados, acometida' de disenteria, cuja etiolojia permanecia obscura; antes desse primeiro exame, a doente fôra improficuamente tratada pelo sulfato de sodio, pós de DOWER, calomelanos, electrargol em lavajens intestinaes e injeções de oleo canforado. No fim de 12 dias de molestia a doente veiu a falecer. A sintomatolojia era constituida principalmente por abatimento, evacuações dolorosas, fezes sanguinolentas, lingua saburrosa, ventre timpanico e doloroso; nos dous primeircs 
dias de molestia, houve lijeira hipertermia (maximo de 370,6 C.), nos dias seguintes a temperatura atinjiu a $38^{\circ}$ a 390 C. á tarde e 370 a $37 \circ, 6$ C. pela manhã ; nos dous ultimos dias de molestia, a temperatura caiu e se manteve a $36^{\circ} \mathrm{C}$. O primeiro exame que fizemos das fezes desta doente foi realizado na vespera de sua morte, o que não nos permitiu fazer o exame bateriolojico das fezes, o qual decidiria do papel etilojico do flajelado.

\section{Genero Chilomitus (') mihi, 1915.}

Chilomitus cavio, mihi, 1915

Descrição. - O flajelado apresenta um dimorfismo muito acentuado, havendo, porém, entre as duas fórmas extremas, muitos aspetos intermediarios. primento, por cerca de $4 \mu$ de largura; 0 corpo é nitidamente deprimido no sentido lonjitudinal; a extremidade anterior é arredondada e larga, a extremidade posterior é afilada, mas não constitue cauda; um dos dous bordos do corpo é mais espesso e mais convexo que o outro; o citostoma é dirijido obliqua ou quasi paralelamente, em relação ao eixo lonjitudinal do corpo, vindo terminar no limite entre a borda mais delgada e a extremidade anterior.

Sob outra fórma, o flajelado é curto, tendo cerca de 8 a $10 \mu$ de comprimento, por 4 a $5 \mu$ de largura; as extremidades são egualmente arredondadas e muito largas; o corpo é fortemente deprimido no sentido lonjitudinal ; uma das bordas é muito espessa, arredondada e ap-osenta grande convexidade; a outra borda é muito delgada, quasi,

Esquema da morfolojia de Chilomitus caviæe

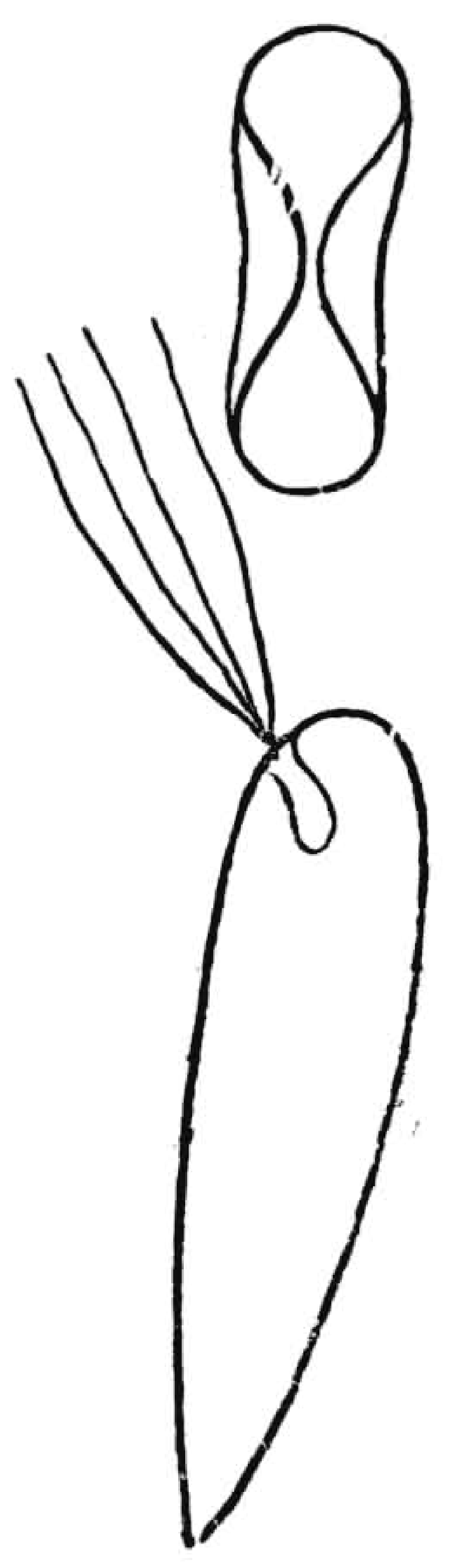

I-Fórma larga, de perfil.

II-Fórma larga, de face.

Sob uma das fórmas, o flajelado se apresenta alongado, com 12 a17 $\mu$ de com-

(1) De $x \varepsilon \tilde{\imath} \lambda o s$, labio + $\mu$ iros, fio.

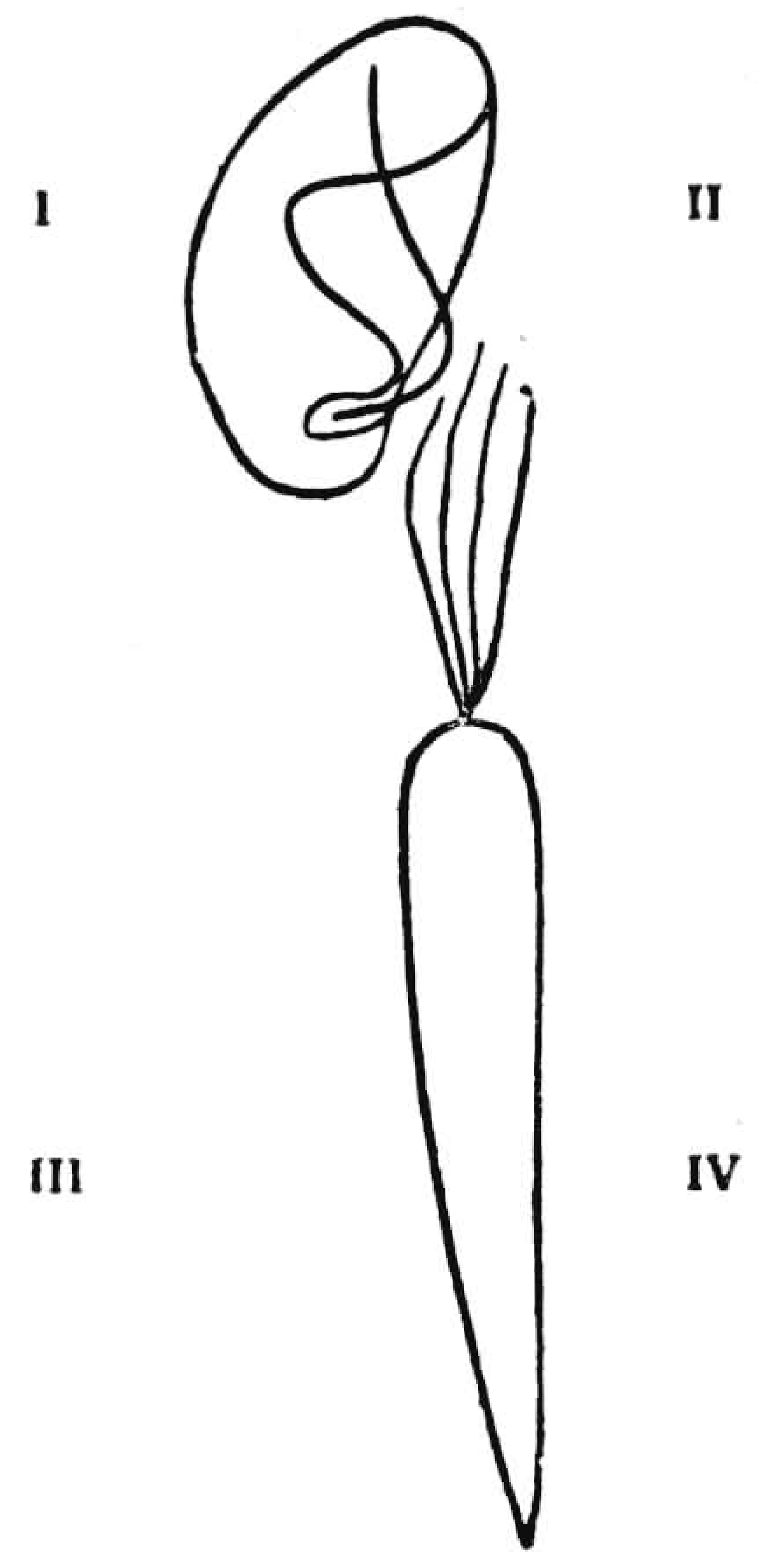

III-Fórma longa, de perfil.

IV-Fórma longa, de face.

laminar e tem convexiâade muito pouco acentuada; o citostoma perpendicularmente dirijido, em relação ao eixo lonjitudinal do corpo, vem terminar na parte mais anterior 
da borda delgada. $O$ limite entre a parte laminar e a parte espessa do corpo, fórma uma curva nitida, com a concavidade dirijida para a borda delgada, e, ás vezes, parece se continuar com a linha limitante do citostoma; é ao lado da concavidade dessa curva que, frequentemente, os flajelos se acham escondidos formando um feixe.

O citostoma tem quasi sempre o aspeto de saco, que ás vezes se estreita junto á abertura; tem 1, a $2 \mu$ no maximo de largura, por cerca de 3 a $4 \mu$ de comprimento.

Plasina distinto em endo e etoplasma. Este apresenta uma camada periplastica espessa, verdadeira capsula, cuja rijidez impede o menor movimento metamorfico, o resto do etcplasma é hialino e se continua com o endoplasma cujas granulações siderofilas, irregulares e numerosas se acumulam, principalmente, nas rejiões centraes do protozoario.

Nucleo dificil de distinguir dentre as numerosas granulações siderofilas; está disposto anteriormente, tem cariosoma volumoso e zona do suco nuclear vasia.

Corpusculo basal tambem dificilmente perceptivel situado proximo ao citostoma, e ligado ao cariosoma do nucleo por meio de um rizoplasto. Deste corpusculo basal, saem para frente, pelo citostoma, quatro flajelos anteriores, todos mais ou menos do tamanho do corpo; nas fórmas largas, ás vezes, eles formam um feixe cuja extremidade livre se vem ocultar na concavidade limitante da borda delgada do parasito.

Habitat. - Céco de Cavia aperea e de C. porcellus.

Familia Hexamitidae KENT, 1880-82.

Sinonimia. - Distomata KLEES, 1892, paj. 329 ; Distomataceo SENN,. 1900, paj. 147; Distomatida HARTMANN e CHAGAS, 1910, paj. 118.

Genero Octomitus (') PROWAZEK, 1904.

Sinonimia. - Dicercomonas GRASSI, 1882 ; Hexamita DUJARDIN, 1838, pro parte;

(2) De $i x \tau \omega$, oito $+\mu i$ iros, fio.
Urophagus MOROFF, 1903, pro parte.

Especies conhecidas:

O. intestinalis (DUJA RDIN, 1841).

O. murıs (GRASSI, 1882).

Octomitus muris (GRASSI, 1882).

Sinonimia. - Dicercomonas muris GRASSI 1882; Hexamitus muris (GRASSI, 1881) auctorum.

Redescrição. - Corpo em fórma de massa ou de bastão aiongado, ás vezes mais ou menos encurvado, de 9 a $10 \mu$ de com. primento por 2 e $4 \mu$ de largura. Extremidade anterior arredondada, romba e mais larga que a posterior que é afilada, mas não ponteaguda.

Plasma distinto em etoplasma, constituido só pela delgada camada periplastica, e endoplasma pouco distintamente alveolado e contendo poucas inclusões; a zona lonjitudinal situada na parte média do corpo é mais clara e acompanhada em seus dous lados por duas linhas cromófilas, dependentes do aparelho locomotor do flajelado e confundidas quasi sempre som os axostilos de varios Tetramitidoe; é para essa formação cromatica, identica á de Giardia s Cercomonas que propomos conservar o nome de rizostilo, reservado jor ALEXEIEFF, para formação analoga dos Rhizomastix; esse ultimo autor justifica a distinção entre axostilo e rizostilo dizendo); "Sa fonction n'est pas analogue à celle de l'axostyle. En effet, ce dernier est surtout destiné à maintenir constante la forme du corps et représente ainsi une formation squelletrque, tandis que le rhizostyle fait partie intégrante de l'appareil flagéllaire." Esses dous rizostilos são paralelos e separam, para fóra, duas zonas mais escuras afiladas posteriormente; nestas ronas existem muitas vezes granulos cromófilos, mais ou menos volumosos e esparsos.

Em contintiação aos rizostilos, saem da extremidade posterior do parasito dous flajelos caudaes delgados $\mathrm{e}$ menores que $o$ corpo.

$\mathrm{Na}$ extremidade anterior das zonas lateraes escuras do corpo, estão os dous nucleos alongados, simetricamente dispostos e for- 
maāos de granulações cromaticas irregulares.

Estes nucleos estăo ligados a dous corpusculos basaes marjinaes, colocados simetricamente na extremidade anterior do protozoario. De cada corpusculo basal, saem, para frente e para um lado, trez flajelos do tamanho dos posteriores.

Habitat. - Intestino de Mus musculus, fórma cinzenta e fórma albina; de Mus norwegicus, fórma cinzenta e albina, e de Mus rattus.

\section{Genero Giardia KUNSTLER, 1882.}

Sinonimia. - Cercomonas DUJARDIN, 1841, in LAMBL, 1859 ; Dimorphus GRASSI, 1879 (nom. preoc. por aracnideos); Hexamitus DUJARDIN, 1841, in DAVAINE, 1875; Lamblia BLANCHARD, 18s8; Megastoma GRASS1, 1881 (nom. creado por BLAINVILLE, para moluscos; por COSTA, em 1850 , para peixes; por SWAINSON, em 1837, para aves; por MEGERLE e MÜHLFELD, para moluscos).

Especies conhecidas:

Giardia agilis KUNSTLER 1882.

G. alata KUNSTLER et GINESTE, 1907.

G. cuniculi (BENSEN, 1908).

G. intestinalis (LAMBL, 1859).

G. microti KOFOID et C.HRISTIANSEN, 1915.

G. muris (BENSEN, 1907).

Morfolojia. - Flajelados em geral piriformes ou claviformes, providos de ventosa discoide que ocupa toda a parte anterior da celula, cuja porção restante se vae progressivamerite afilando, até terminar em um prolongamento caudal flexivel.

Plasma constituido de periplasta resistente que sósinho representa todo o etoplasma, e endoplasma de estrutura mais ou menos granular, desprovido de granulações e de alveolos. Não ha movimentos metamorficos.

O plasma é separado por filamentos cromófilos em rejiões diferentes pela espessura e colorabilidade que apresentam.

Dous nucleos com cariosoma central cercado de zona do suco nuclear vasia; membrana nuclear espessa e fortemente cr mófila. No centro do cariosoma existe, ás vezes, centriolo cromatico; outras vezes, se observam duas a quatro pequenas granulações de cromatina. No polo anterior de cada nucleo, existe uma granulação cromatica bariliforme, aderente á membrana nuclear e ligada ao cariosoma por meio de fino rizoplasto.

Adeante do espaço intermediario entre os dous nucleos, existe, formando um arco de concavidade anterior, um grupo de quatro granulos cromaticos perfeitamente identicos aos corpusculos basaes; esses granulos estão ligados entre si e ás granulações bacilares da membrana nuclear, por meio de rizoplasto. Dos dous granuios externos desse grupo, saem dous filamentos que se encurvam para deante de modo a virem se cruzar na linha média ; a curvatura continúa até que o filamento encontra a superficie da celula, no ponto mais lateral da extremidade anterior; nessa ocasião, os dous filamentos soltam-se para o exterior, como flajelos livres. Dos dous granulos internos do grupo. saem dous filamentos cromaticos que caminham retus e paralelos até a extremidade posterior, donde tambem se continuam como dous flajelos caudaes; toram esses dous filamentos axiaes do parasito que, como os dos Octomitus, varios autores confundiram com $o$ axostilo tipico dos Trichomonas e Trichomastix; a esses do!ıs filamentos, pelas mesmas razões que expuzemos para o caso dos Octomitus, deve caber a denominição de rızostilos. Dos mesmos granulos donde partem os rizostilos saem, para cada lado, dous outros filamentos; de cada lado um deles se encurva para fóra e depois para deante, de modo a descreverem um ovoide em torno de cada nucleo e se terminarem no ponto de emerjencia dos flajelos anteriores; os outros dous filamentos caminham lijeiramente encurvados para fóra e para traz e terminam na superficie do parasito, ao nivel da união do terço médio com o terço posterior, ponto em que se continuam livres no exlerior como flajelos lateraes. Na parte média de cada rizostilo existe uma granulação, cromatica baciliforme, donde emerjem dous flajelos medianos e livres que, quasi sempre, caminham paralelos para um lado. descrevendo duas 
curvaturas que dão a cada um deles a conformação de um $S$ italico.

Pouco para traz do ponto de emerjencia dos flajelos, existe um corpo cromófilo de natureza cromidial, cuja fórma serve de carater especifico e cujo papel fisiolojico é totalmente ignorado.

Giardia cunıculi (BENSEN, 1908).

Sinonimia. - Cercomonas intestinalis LAMBL, 1859, pro parte; Hexamitus duodenalis DAVAINE, 1879, pro parte; Lamblia intestinalis BLANCHARD, 1880, pro parte; Lamblia cuniculi BENSEN, 1908; Megastoma entericum GRASSI, 1881; Megastoma intestinalis BLANCHARD, 1886.

Descrição. - Corpo com 10 a $20 \mu$ de comprimento por 6 a $12 \mu$ de largura, formado de grande parte anterior discoide que se continua para traz pelo prolongamento caudal que começa meio bruscamente ao nivel do equador do disco anterior.

Nucleos ovoides e cariosomicos; cariosomas frequentes vezes com diversas granulações cromaticas em seu interior. Corpusculos basaes do grupo quadruplo anterior, bastante afastados uns dos outros, formando arco de curvatura pouco pronunciada.

Cromidio alongado, perpendicular ou obliquo em relação ao eixo do animal; ás vezes é triangular, outras vezes é constituido por duas barras cromaticas mais ou menos encurvadas e ligadas entre si por uma extremidade; geralmente lateral ; esse cromidio se distingue por esse fato dos das especies seguintes.

Flajelos eguaes, delgados, mais ou menos com comprimento egual a metade do tamanho do corpo.

Habitat. - Intestino delgado de Coendu villosus e de Oryctolagus cuniculus.

Giardia intestinalis (LAMBL, 1859).

Sinonimia. - Cercomonas intestinalis LAMBL, 1859, pro parte; Hexamitus duodenalis DAVAINE, 1875, pro parte; Lamblia intestinalis, BLANCHARD, 1888, pro parte; Megastoma enterıcum ORASSI, 1881, pro parte; Megastoma intestinale BLANCHARD, 1886, pro parte.

Descrição. - Dimensōes variaveis e identicas ás da especie precedente; fórma do corpo analoga; apenas o afilamento do parasito para a parte posterior se faz menos bruscaniente; extremidade caudal menos afilada.

Nucleos ovoides, cariosomicos; cariosomas frequentemente ligados por meio de rizoplastos aos corpusculos basaes; estes estão no grupo quadruplo, formando arco de cencavidade posterior e de curvatura pouco acentuada. A's vezes, não existe cariosoma e a cromatina se acha aderente á membrana nuclear, geralmente no pólo posterior do nucleo, onde constitue uma barra em crecente.

Cromidio em bastonete, perpendicularmente disposto em relação ao rizostilo, geralmente mediano, raramente um tanto lateral, o que faz confundir ás vezes este flajelado com o precedente.

Flajelos mais ou menos do tamarho de metade do comprimento do corpo.

Habitat. - Intestino de Homo sapiens e de Cebus caraiá.

Giardia muris (BENSEN, 1908).

Sinonimia. - Cercomonas intestinalis LAMBL, 1859, pro parte; Dimorphus muris GRASSI, 1879; Hexamitus duodenalis DAVAINE, 1875; Lamblia intestinalis BLANCHARD, 1888; Lamblia muris BENSEN, 1908; Megastoma entericum GRASSI, 1881; Megastoma intestinale BLANC.HARD 1886.

Descrição. - Dimensões variam como nas especies precedentes, sendo, porém, o corpo mais largo e mais curto na $G$. muris que nas outras especies do genero. Ventosa anterior relativamente maior, afilamento posterior mais brusco, extremidade caudal quasi ponteaguda e mais bruscamente formada.

Nucleos mais arredondados que nas especies precedentes, ora cariosomicos, ora com cromatina aderente a um dos polos.

Os corpusculos basaes formam um grupo em linha fortemente encurvada, de concavidade posterior. 

dentes.

Flajelos curtos como nas especies prece-

Habitat. - Intestino delgado de Mus norwegicus.

\section{Anexo}

Genero Selenomonas (') PROWAZEK, 1913.

Sinonimia. - Ancyromonas KENT, 188082. Selenomastix WOODCOCK et LAPAGE, 1913.

Selenomonas ruminantium (CERTES, 1889).

Sinonimia. - Ancyromonas ruminantium CERTES, 1889; Selenomastix ruminantium WOODCOCK et LAPAGE, 1913.

Historico. - Em 1889, CERTES colocou no genero Ancyromonas de KENT um interessante protista que ulteriormente PROWAZEK verificou dever ser separado do genero $A n c y$ romonas para constituir novo genero, cujas relações de semelhança com Spirillum sputigenum o mesmo autor verificou.

Descrição. -- Parasito em fórma ae crecente com dimensões muitissimo variaveis, frequentemente entre 8 a $20 \quad \mu$ de comprimento, por cerca de $3 \mu$ de largura.

Espessa e rijida membrana celular, com caracteres fisicos da celulose, envolve toda a celula. Plasma de estrutura alveolar dificilmente verificavel.

Cromatina ás vezes esparsa pelo plas ma, outras vezes condensada em uma granulação cromatica, ás vezes dupla, disposta junto á membrana celular, na concavidade do crecente.

(1) De $\sigma \varepsilon \lambda \eta \dot{v} \nu \eta$, lua $+\mu o v a ́ s$, unidade.

Dessa granulação cromatica, parte um espesso flajelo mais ou menos do comprimento do corpo.

A's vezes o flajelo é duplo ou multiplo no primeiro caso a interpretação verosimil é de estar o fato relacionado com a divisão transversa habitual no protista. No 'segundo caso parece tratar-se de fenomenos dejenerativos.

Habitat. - Céco de Cavia aperea, C. porcellus e Dasyprocta aguti.

Nota. - O parasito é descrito como encontrado no estomago dos ruminantes domesticos e selvajens (CERTES, KERANDEL, DOMIZIO, PROWAZEK) nos glanglios linfaticos de cobaio (SPLENDORE), no sangue de falcăo, etc. Foi ele pela primeira vez encontrado nos trez roedores que citámos pelo Dr. ARISTIDES MARQUES DA CUNHA o qual sobre o asunto havia publicado, em 1915, uma nota prévia. Alguns autores descreveram como fase evolutiva de Selenomonas outros protistas do estomago de ruminantes: pequenas celulas redondas, sem organs locomotores aparentes e de ex. trema mobilidade. Parece não dever prevalecer esta opinião.

Nenhuma certeza existe si o parasito em questão é ou não um flajelado; o nucleo individualizado, o flajelo espesso parecem corroborar essa conclusão; a presença, porém, de membrana celular tão semelhante á dos vejetaes, a divisão transversa do protista deixam muitas duvidas, sobre a conclusão a tirar. No caso de ser um flajelado, a que ordem deveiia pertencer? So por grande concessão poderia ser incluido, no sistema de HARTMANN, entre as rizomastijinas. 


\section{$-30-$ \\ Bibliografia.}

ALEXEIEFF, A.

ALEXEIEFF, A.

ALEXEIEFF, A.

ALEXEIEFF, A.

ALEXEIEFF, A.

ALEXEIEFF, A.

ALEXEIEFF, A.

ALEXEIEFF, A.

ALEXEIEFF, A.

ALEXEIEFF, A.

ALEXEIEFF, A.

ALEXEIEFF, A.

ALEXEIEFF, A.

ALEXEIEFF, A.
1908 Sur la division de Hexamitus intestinalis Duj. Compt. rend. de la Soc. de Biol. An. 60, pp. 402-404, 1 fig. Paris.

1909 Les flagellés parasites des batraciens indigènes. Compt. rend. de la Soc. de Biol. An. 61, t. 2, pp. 199-201 Paris.

1909 Un nouveau Trichomonas à quatre flagelles antérieurs. Compt. rend. de la Soc. de Biol. An. 61, pp. 712-714. Paris.

1910 Sur les flagellés intestinaux des poissons marins (note préliminaire). Arch. Zool. expér. et généraie. 5e série. Vol. 6. Notes et révue, pp. I-XX, 12 fig. Paris.

1911 Haplomitose chez les Eugleniens et dans d'autres groupes de Protozoaires. Compt. rend. de la Soc. de Biol. An. 63, t. 2, pp. 614.617, 8 fig. Paris.

1911 Quelques flagellés intestinaux rouveaux ou peu connus. Arch. Zool. expér. et générale. 5e série. Vol. 6, pp. 491527 Paris

1911 Sur la famille Cercomonadina Bütschli emend. (non Cercomo. nadida Kent). Compt. rend. de la Soc. de Biol. An. 63, t. 2, pp. 506-508, 6 fig. Paris.

1911 Sur la nature des formations dites «Kistes de Trichomonas intestinalis». Compt. rend. de la Soc. de Biol. An. 63, t. 2, pp. 296-298, 1 fig. Paris.

1911 Sur la position des Monadidés dans la systématique des flagellés. Quelques observations sur le Monas vulgaris. Signification des blépharoplastes. Bul. de la Soc. Zool. France. Vol. 36 pp. 96-103.

1911 Sur la spécification dans le genre Trichomonas Donné. Compt. rend. de la Soc. de Biol. An. 63, t. 2, pp. 539. 541. Paris.

1911 Sur les "Kystes de Trichomonas intestinalis" dans l'intestin des Batraciens. Bul. Scient. de France et Belg. 7e série. Vol. 44, pp. 333-355, 1 est., 2 fig. Paris.

1912 Sur quelques noms de genres des flagellés qui doivent disparaitre de la nomenclature pour cause de synonimie ou pour toute autre raison. Diagnoses de quelques genres récemment étudiés. Zool. Anzeiger. Vol. 39, pp. 674-680, 2 fig. Paris.

1912 Sur quelques protistes parasites d'une tortue de Ceylan (Nicoria trijuga). Zool. Anzeiger. Vol. 40, pp. 97 105, 2 fig. Cf. pp. 97-102.

1913 Systématisation de mitose dite "primitive". Sur la question du centriole (A propos de la division nucléaire chez Malpighiella sp.) Arch. f. Protistenkunde. Vol. 29, pp. 344-363, 7 fig. 
ALEXEIEFF, A.

APSTEIN, C.

ARTAULT, STEPHEN

ASSMY

BENSEN, W.

BENSEN, W.

BENSEN, W.

BERLINER, E.

BILAND, I.

BLANCHARD, $\mathbf{R}$.

BLOCHMANN, F.

BOHNE, A. und PROWAZEK, S. VON

BRAUN, $M$.

BRAUNE, ROBERT

BROWN, W. CARNEGIE 1910

BRUMPT, E.

BRUMPT, E.

BRUMPT, E.

BRUMPT, E.

BÜTSCHLI, O.
1914 Notes protistologiques. Zool. Anzeiger. Vol. 44, pp. 193-213, 5 fig. Cf. pp. 197-200 e 203-213.

1915 Nomina conservanda. Sitzungsber. d. Gesellsch. Nat. Freunde zu Berlin. N. 5, de 5-1915, pp. 119-202. Cf. pag. 192.

1898 Flore et faune des cavernes pulmonaires. Arch. de parasitologie. Vol. 1, pp. 217-307. Cf. pp. 277-28ı, 3 fig. Paris.

1914 Zur Frage der Emetinbehandlung der Lamblienruhr. Münch. med. Wochenschr. Vol. 61, pag. 1393.

1908 Bau und arten der Gattung Lamblia. Zeitschr. f. Hyg. und Infectionskr. Vol. 61, pp. 109-114, 6 fig.

1909 Die Darmprotozoen des Menschen. Arch. f. Schiffs und Trop. Hyg. Vol. 12, pp. 661-676, 7 fig.

1910 Untersuchungen übcr Trichomonas intestinalis und vaginalis des Menschen. Arch. f. Protistenkunde. Vol. 18, pp. 115-127, 3 est. Berlin.

1909 Flagellatenstudien. Arch. f. Protistenkunde. Vol. 15, pp. 297325, 2 est.

1905 Beitrag zur Frage der Pathogenität der Flagellaten. Deutsch. Arch. f. klin. Medizin. Vol. 86, pp. 275-- 993,2 est. Leipzig.

1888 Remarques sur le Mégastome intestinal (Lambla intestinalis, nom. nov.) Bul. de la Soc. Zool. France. Vol. 13, pp. 18-19. Paris.

1884 Bemerkungen über einige Flagellaten. Zeitschr. f. wiss. Zoologie. Vol. 40, pp. 42-49, 1 est. Heidelberg.

Zur Frage der Flagellatendysenterie. Arch. f. Protistenkunde Vol. 12, pp. 1-8, 1 est. , 3 lig.

1908 Die thierischen Parasiten des Menschen. 4a ed. Würzburg. Cf. pp. 51-60, 8 fig.

1913 Untersuchungen über die im Wiederkäuermagen, vorkommenden Protozoen. Arch. f. Protistenkunde. Vol. 32, pp. 111-170. Cf. pp. 119-130, 1 est.

Amebic or tropical Dysentery, its complications and treatment. London. Cf. pp. 82-84, 1 fig.

1909 Bul. de la Soc. Path. éxot. Vol. 2, pag. 20 (Nota sobre casos de infeção de macacos por Trichomonas e Amaeba).

1912 Côlite à Tetramitus Mcsnili (Wenyon, 1910) et côlite à Trichomonas intestinalis Leuckart, 1879. Blastocystis hominis, n. sp. et formes voisines. Bul. de la Soc. Path. éxot. Vol. 5, pp. 725-730.

1913 Précis de Parasitologie. 2a ed. Paris. Cf. pp. 191-200, illustr. 1913 Um caso de enterite á Lamblia intestinalis. An. paul. Med. e Cirurgia. Vol. 1, pp. 67-69, 3 fig. S. Paulo.

1878 Beiträge zur Kenntniss der Flagellaten und verwandte Organismen Zeitschr. f. wiss. Zoologie. Vol. 30, pp. 205-281, 5 est. Carlsruhe. 
BÜTSCHLI, O. 1883-1887 Protozoa. In Bronn, Klassen und Ordnung des Thier Reichs

CAHEN, EUGEN

CASTELlANI, ALDO

CASTEllani, ALDO

CASTELLANI, ALDO

CASTELLANI, ALDO and CHALMERS, ALBERT G.

CASTELLANI, ALDO and CHALMERS, ALBERT $F$.

CHATTERJEE, F. C.

CHATTERJEE, F. C

CHATTON.

CONHEIM, $\mathbf{P}$.

CONHEIM, P.

CUNHA, Dr. ARISTIDES MARQUES DA 1915

CUNHA, Drs. ARISTIDES MARQUES DA e TORRES, MAGARINOS 1914

CUNNINGHAM, D. D. 1881

DARLING

DAVAINE, C.

1910

1913
Vol. 1, T. 2. Cf. Flagellata, pp. 620-876, 9 est.

1891 Ueber Protozoen im kindlichen Stuhle. Deutsch. med. Wochenschr. An. 17, pp. 853-854, 1 fig.

1905 Diarrhœa from Flagellates. Brit. med. Journal. An. 1905, t. 2, pp. 1285-1287, 2 fig. Colombo, Ceylon.

1905 Diarrhoea from Flagellates. Lancet, Vol. 169, pag. 540. Colombo.

1905 Observations on some protozoa found in human foeces. Cbl. f. Bakteriologie, 1. Orig., Vol. 38, pp. 66-69, 5 fig. Cf. pp. 67-69.

Note on an intestinal flagellate in man. The Pnilippine J. of Science. Vol. 5, pp. 211-212, 1 est.

Manual of Tropical Medicine. 2a ed. London. Cf. pp. 280416. Illustr.

1915 On a five flagellate Trichomonas (n. sp.), parasitic in man. Ind. med. Gazette. Vol. 50, pp. 57, 1 est.

1915 On a Macrostoma found in human intestinal contents. Ind. med. Gazette. Vol. 50, pp. 135-136, 1 est.

1912 Bul. de la Soc. Path. éxot. Vol. 5, pag. 499. Paris (Nota á comunicação de $M$. Nattan Larier sobre Tetramitus mesnili).

1909 Ueber Infusorien in Magen und im Darmcanale des Menschen und ihre klinische Bedeutung. Deutsch. med. Wochenschr. Vol 29, pp. 206-208, 230-232 e 245248,1 fig.

1909 Infusorien bei gut. u. bösartigen Magenleiden nebst Bemerkungen über sogenannte Infusorienenteritides. Deutsch. med. Wischenschr. An. 1909, pp. 92-95.

Sobre a presença de Selenomonas no cœcum dos roedores. Braz. medico, N. 5 de 1915. (1 de Fevereiro). Rio de Janeiro.

Sobre alguns casos de colite produzida pelo Chilomastix mesnili (Wenyon, 1910). Braz. medico. N. 28 de 1914 (22 de Julho), Rio de Janeiro.

On the development of certain microscopical organisms occurring in the intestinal canal. Quart. J. of microsc. Science. Vol. 21, pp. 234-290, 1 est.

1909 An infection by Lamblia intestinalis in an American child. Proc. of the Canal Zone Med. Assoc. Fasc. 44, pag. 120.

1845 Sur les animalcules infusoires trouvés dans les selles de malades atteints du choléra et d'autres maladies. 
Compt. rend. de la Soc. de Biol. 2e série. Vol. 1, pp. 129-130.

DAVAINE, C.

DAVAINE. C.

DAVISON, ANDREW

DÉLAGE, YVES et HÉROUARD, EDGARD 1896

DERIEU et RAYNAUD 1914

DOBELL, C. CLIFFORD 1907

DOBELL, C. CLIFFORD 1909

DOBELL, C. CLIFFORD 1910

DOFLEIN, F.

DOFLEIN, F.

DUJARDIN, $F$.

DUJARDIN, $F$.

EHRENBERG, CRISTIAN GOTTFRIED 1838

ELLERMANN, V.

ESCOMEL, P.

FAIRISE, C. et

JANNIN, L.

1913

FICKER, MARTIN

FISCHER, C.

FLU, P. C.

FOA, ANNA

1875

1877

1909

1907

\section{Trai}
Dysentérie chronique à flagellé nouveas. Bul. de la Soc. Path. éxot. Vol. 7, pp. 571-574.

Trichomastix serpentis, n. sp. Quart. J. of microsc. Science. Vol. 51, pp. 449-458, 1 est. 2 fig.

Researches on the intestinal protozoa of frogs and toads. Quart. J. of microsc. Science. Vol. 53, pp. 201279. Cf. pp. 206-245.

On some parasitic protozoa from Ceylon. Spolia Zeylanica. Vol. 3, pp. 65-87, 1 est. Colombo, Ceylon.

1902 Das System der Protozoen. Arch. f. Protistenkunde. Vol. 1, pp. 159-192. München.

1911 Lehrbuch der Protozoenkunde. Jena. Cf. pp. 382-386 e 471505.

1838 Sur les Monades à filament multiple. Ann. Sci. naturelles. Vol. 10 , pp. $17-20$.

1841 Histoire naturelle des Zoophytes Infusoires. Paris.

Die Infusionsthierchen als volkommene Organismen. Berlin und Leipzig.

Über kieinste Mikroorganismen im menschlichen Speichel. Cbl. f. Bakteriologie. P. 1. Orig: Vol. 44, pp. 160164, 3 fig. Kopenhagen.

1913 Sur la dysentérie à Arequipa (Pérou). Bul. de la Soc. Path. éxot. Vol. 6, pp. 120-122.

Dysentérie chronique à «Lamblia». Etude parasitologique et anatomo-pathologique. Arch. de Méd. expér. et d'Anat. pathologique. Vol. 25, pp. 525-551, 5 fig. Nancy.

1915 Sobre a dysenteria em São Paulo. Ann. paul. Med. e Cirurgia. Vol. 5 (Livio do Jubileu do Dr. Luiz Pereira Barreto)

1885 Untersuchungen über einige Flagellaten und verwandte Organismen. Zeitschr. f. wiss. Zoologie. Vol. 42, pp. 47-125, 4 est. Erlenger.

1908 Uber die Flagellaten in Darm von Melophagus ovinus. Arch. f. Protistenkunde. Vol. 12, ip. 147-153.

1904 Ricerche intorno a due specie (Dicercomonas muris Grassi e D. intestinalis Duj.) di flageilati parassiti. Rendiconti Acad. dei Lincei. Serie 5. Vol. 13, 10 sem. de 1904, pp. 121-130. Roma. 


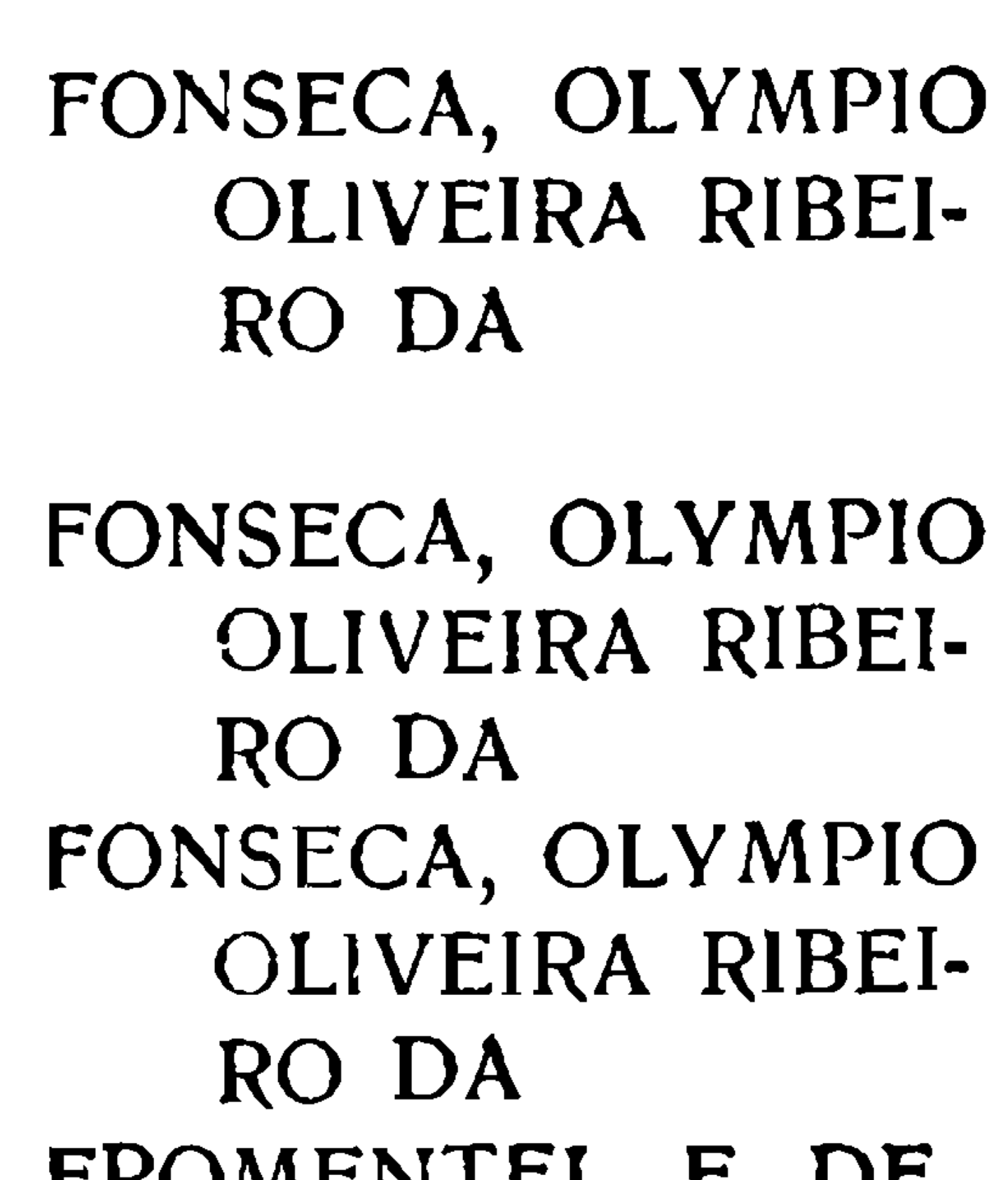

GÄBEL, MAX

GALLI, VALERIO, B.

GALLI, VALERIO, B.

GINESTE, CH.

GINESTE, CH.

GINESTE, $\mathrm{CH}$.

GONDER, RICHARD

ORASSI, BATTISTA

GRASSI, BATTISTA

GRASSI, BATTISTA

GRASSI, BATTISTA

GRASSI, B. und SCHEWIAKOFF, W. 1915 1915

1914 Zur Pathogenität der Flagellaten. Ein Fall von Tetramitidendiarrhœe. Arch. f. Protistenkunde. Vol. 34, pp. 134, 2 est.

1900 Notes de parasitologie. Cbl. f. Bakteriologie. Vol. 27, pp. 305309.

1907 Notes de parasitologie. B. Parasites animaux. Cbl. f. Bakteriologie. P. 1. Orig. Vol. 44, pp. 523-532. Cf. pp. 528-529, 1 fig. Lausanne.

1911 Mouvements amiboides et ondulatoires chez les infusoires flagellés. Compt. rend. de la Soc. de Biol. An. 63, t. 1, pp. 1014-1016. Bordeaux.

1913 Chromidies et dıalité nucléaire chez les flagellés. Compt. rend. de la Soc. de Bíol. An. 65, pp. 405-408.

1913 L' " appareil nucléaire» de quelques Cercomonades. Compt. rend. de la Soc. de Biol. An. 65, pp. 408-410.

1911 Lamblia sanguinis, n. sp. (Gonder). Arch. f. Protistenkunde. Vol. 21, pp. 208-212, 1 fig. Transvaal.

1881 Ad alcuni protisti endoparassitici et appartenenti alle classi dei Flagellati, Lobosi, Sporozoi e Ciliati. Cf. pp. 10-47, 2 est. Rovellasca.

1888 Les protozoaires parasites de l'homme. Arch. ital. de Biol. Vol. 9 , pp. 4-6.

1888 Morfologia e sistematica di alcuni protozoi parassiti. Rendiconti Acad. dei Lincei. $4^{a}$ serie, 10 sem. de 1888, pp. 5-12 Roma.

1888 Significato patologico dei protozoi parassiti dell'uomo. Rendiconti Acad. dei Lincei. 4 a serie. 10 sem. de 1888, pp. 83-89.

Beitrag zur Kenntniss des Megastoma entericum. Zeitschr. f. wiss. Zoologie. Vol. 46, pp. 143-154, 1 est.

GRUBY et DELAFOND 1843 Recherches sur les animalcules se développant dans l'estomac et dans les intestins des animaux herbivores et carnivores. Compt. rend. de l'Acad. de Sc. Vol. 17 , pp. 1304-1308.

HARTMANN, MAX
1907 System der Protozoen. Arch. f. Protistenkunde. Vol. 10, pp. 139-158, 3 fig. 
HARTMANN, MAX

HARTMANN, MAX e CHAGAS, CARLOS 1910

JANICKI

JANICKI

JOLLOS, VICTOR

KANNENBERG

KENT, SAVILLE KERANDEL, J.

KLEBS, $\mathbf{O}$.

KOFOID, CHARLES ATWOOD and CHRISTIANSEN, ELIZABETH B.

KUCZYNSKI,

KUNSTLER, J.

KUNSTLER, J.

KUNSTLER, J.

KUNSTLER, J. et GINESTE, $\mathrm{CH}$.

KUNSTLER, J. et GINESTE, CH.

LAVERAN et MESNIL

LEITÃO, Dr. MELLO 1896 1907

1915

19

1882

1883

1896

1901
1912 System der Protozoen. In Prowazek, Handbuch der Pathogenen Protozoen. Cf. pp. 41-49.

Estudos sobre flagellados. Mem. do Inst. Osw. Cruz, Vol. 2, fasc. 1, pp. 64-125, 6 est. 3 fig. Rio de Janeiro. 1910 Untersuchungen an parasitischen Flagellaten. Teil I. Zeitschr. f. wiss. Zoologie. Vol. 95, pp. 243-315, 16 fig., 4 est. Basel.

1911 Zur Kenntnis der Parabasalapparates bei parasitischen Flagellaten. Biol. Cbl. Vol. 31, pp. 321-330, 8 fig.

1911 Studien über parasitische Flagellaten I. Monocercomonas cetoniae, n. sp. Arch. f. Protistenkunde. Vol. 23. pp. 311-318, 1 est. München.

Ueber Infusorien im Sputum. Arch. f. pathol. Anat. u. Physiologie. Vol. 65, pp. 471-474, 2 fig.

1880-82 A manual of Infusoria. London.

1909 Sur quelques hématozoaires observés au Congo (Haute SanghaLugone) Bul. de la Soc. Path. éxot. Vol. 2, pp. 204-209. Cf. pag. 208.

1892 Flagellatenstudien. Theil I. Zeitschr. f. wiss. Zoologie. Vol. 55, pp. 265-351, 4 est.

Theil Il. Zeitschr. f. wiss. Zoologie. Vol. 55, pp. 353-445, 2 est. Basel.

On the life history of Giardia. Proc. of the National Acad. of Sciences. Vol. 1, pp. 547-552, 1 fig.

Untersuchungen an Trichomonaden. Arch. f. Protistenkunde. Vol. 33, pp. 119-204, 6 est., 4 fig. Rostock.

Sur cinq Protozoaires parasites nouveaux. Compt. rend. de l'Acad. des Sc. Vol. 95, pp. 347-349. Paris.

Recherches sur les infusoires parasites. Sur quinze Protozoaires nouveaux. Compt. rend. de l'Acad. des Sc. Vol. 97, pp. 755-757. Paris.

Recherches sur la morphologie du Trichomonas intestinalis. Compt. rend. de l'Acad. des Sc. Vol. 123, pp. 839-842. Paris.

Contribution à la morpholugie des Protozoaires supérieurs. Compt. rend. de l'Acad. des Sc. Vol. 142, pp. 294-296. Paris.

Giardia alata (nov. spec.). Compt. rend. de l'Acad. des Sc. Vol. 144, pp. 441-443, 1 fig. Paris.

Sur la morphologie et la systématique des flagellés à membrane ondulante (genres Trypanosoma Gruby et Trichomonas Donné). Compt. rend. de l'Acad. des Sc. Vol. 133, pp. 131-137, 5 fig. Paris.

1912 Importancia dos flagellados nas dysenterias da infuncia. Arch. brasileiros de Med. An. 2, n. 5, pp. 582-590. Rio de Janeiro. 
LEMMERMANN, E. 1910 Flagellatae In Kryptogamenflora der Mark Brandenburg. Vol. 3 (Algen I) pp. 257-562. Illustr. Leipzig.

LEMMERMANN, E. 1913 Notizen über Flagellaten. Arch. f. Hydrobiol. und Planktonkunde. Vol. 8, pp. 555-574 Cf. 559-561 e 566-571. Bremen.

LEMMERMANN, E. 1914 Flagellate In Pascher, Süsswasserflora Deutschlands, Osterreichs u. d. Schweiz. P. 1, fasc. 1: Pantostomatinae, Protomastiginae, Distomatinae. Cf. pp. 28 138. Illustr.

LEUCKART

LIEBETANZ, ERWIN 1910

LINNCEUS, CAROLUS 1758

MACKINNON, DORIS L. 1910

MACKINNON, DORIS L. 1912

MACKINNON, DORIS L. 1913

MACKINNON, DORIS L. 1913

MACKINNON, DORIS L. 1914

MARCHAND, F.

MARCHAND, F.

MARCHAND, F.

MARTIN, C. H. and ROBERTSON, $M$. MATHIS, C.

MATHIS, C.

MAY, RICHARD

MAYER, MARTIN

METZNER, R.

MEYER, $H$.

1875

1894

1893

1911

1913

1914

1892
1914 Beitrag zur Emetinbehandlung der Ruhr. Die Wirkung des Emetins bei der Lamblienruhr. Münch. med. Wochenschr. An. 61, pp. 241-242. Hamburg.

1902 Untersuchungen au Megastoma entericum Grassi aus dem Kaninchendarm. Zeitschr. f. wiss. Zoologie. Vol. 70, pp. 299-320, 1 est. Basel.

1897 Untersuchungen über einige Flagellaten. Rév. Suisse Zool. Vol. 5, pp. 43-89, 2 est. 
MILLONS.

MINCHIN, E. A.

MINCHIN, E. A.

MIURA, K.

MORISON, J.

MORITZ, F. u. HOLZL, $\mathrm{H}$. 1893

MOROFF, THEODOR

NATTAN-LARIER, $L$.

NOC. F.

OliVEIRA, Dr. OLINTHO

PARISI, BRUNO

PERRONCITO, E.

PERRONCITO, E.

PERRONCITO, E.

PERRONCITO, E.

PERRONCITO, E.

PERRONCITO, E.

PERTY. M.

POENARU, I.

PROWAZEK, S. V.
1913 Remarques sur des cas de Dysentérie à Thanh hoa, en 1912.

Bul. de la Soc. Med. Chirurg. de l'Indochine. Vol. 4, pp. 7-13.

1909 Protozoa In Allbut a. Rolleston, A system of medicine. Vol. 2, p. 2, pp. 9-122. Cf. pp. 55-56.

1912 Introduction to the study of Protozoa, with special reference to the parasitic forms. London. Cf. pp. 257-322.

1894 Trichomonas vaginalis im freschgelassenen Urin eines Mannes. Cbl. f. Bakteriologie. Vol. 16, pp. 63-73, 2 fig. Tokio.

1915 The causes of Monsoon diarrhea and dysentery in Poona. Second report. The Ind. J. of Med. Research. Vol. 2, pp. 950-976. Cf. pag. 953.

Uber Häufigkeit u. Bedeutung des Vorkommens von Megastoma entericum im Darmcanai des Menschen. Münsh. med. Wochenschr. Vol. 39, pag. 76.

1904 Beitrage zur Kenntniss einiger Flagellaten. Arch. f. Protistenkunde. Vol. 3, pp. 69-100, 1 fig., 2 est. München.

1912 Infection humaine due à Tetramitus Mesnili. Bul. de la Soc. Path. éxot. Vol. 5, pp. 495-499, 1 est. Paris.

1909 Le cycle évolutif de Lamblia intestinalis. Bul. de la Soc. de Path. éxot. Vol. 2, pp. 93-97, 1 fig. Saigon e Paris.

A dysenteria amebica na infancia. Braz. med. An. XVIII, nos 32-35, pp. 321-323, 331-334, 341-345. Cf. pag. 332.

1910 Su alcuni flagellati endoparassitici. Arch. f. Protistenkunde. Vol. 19, pp. 232-239, 1 est. Milano.

1887 Ueber die Einkapselung des Megastoma intestinalis. Cbl. f. Bakteriologie. Vol. 2, pp. 738-739. Turin.

1888 Encapsulement du Mégastoma intestinal. Arch. ital. de Biol. Vol. 9, pp. 165-167.

1888 Note sur l'enkystement du Mégastoma intestinal. Bul. de la Soc. Zool. France. Vol. 13, pp. 16-18. Turin.

1888 Ueber die Art der Verbreitung des Cercomonas intestinalis. Cbl. f. Bakteriologie. Vol. 4, pp. 220-221.'Turin.

1901 I parassiti dell'uomo e degli animali utili e la piú comuni mallatie da essi prodotte. Milano.

1902 Une maladie mortelle du Lapin produite par la Lamblia intestinalis de l'homme et du rat. Bul. de la Soc. Zool. France. Vol. 27, pp, 151-155, 1 fig.

1852 Zur Kenntniss kleinter Lebensformen nach Bau, Functionen, Sistematik, etc. Bern.

1911 Sur un flagellé rencontré dans une éruption vulvo-vaginale pustulo-ulcereuse, chez une bufflesse. Compt. rend. de la Soc. de Biol. An. 63, t. 1, pp. 624-625. Paris."

1899 Kleine Protozoenbeobachtung. Zool. Anzeiger. Vol. 22, pp. 339-345, 1 fig. Cf. pag. 335. Karlsdorf. 
PROWAZEK, S. V. 1902 Notiz über Trichomonas hominis. Arch. f. Protistenkunde. Vol.

PROWAZEK, S. V. 1903 Flagellatenstudien. Arch. f. Protistenkunde. Vol. 2, pp. 195-212. Wien.

PROWAZEK, S. V. 1904 Untersuchungen über einige parasitische Flagellaten. Arb. aus d. Kaiserl. Oesundheitsamte. Vol. 21, pp. 1-41, 4 est.

PROWAZEK, S. V. 1911 Zur Kenntniss der Flagellaten des Darmtraktus. Arch. f. Protistenkunde. Vol. 23, pp. 96-100, 16 fig. Hamburg.

PROWAZEK, S. V. 1912 Beiträge zur Kenntniss der Protozoen und verwandte Organismen von Sumatra (Deli). VII. Arch. f. Protistenkunde. Vol. 26, pp. 250-274, 3 est., 1 fig. Cf. pp. 253-255.

PROWAZEK, S. V. 1913 Zur Parasitologie von Westafrica. Cbl. f. Bakteriologie. Orig. P. 1. Vol. 70, pp. 32-36, 1 est. Cf. pp. 35-36.

PROWAZEK, S. V. u. WERNER, $\mathrm{H}$.

1914

RATZ, STEPHEN V. 1913

ROOS, ERNST

1893

ROOS, ERNST

ROSENFELD, ARTHUR 1904

ROUBAUD, E.

SCANZONI, F. W.

1858

SCANZONI, F. W. DE et KÖLLIKER, A. 1855

SCHMIDT, A.

1895

SCHRÖDER, B.

1903

SCHÜRMAYER, B.

SENN, F.

STEIN, F. RITTER VON 1878
1900 Flagellata In Engler u. Prantl, Naturlichen Pflanzenfamilien P.

Zur Kenntniss der sog. Flagellaten. Arch. f. Schiffs u. Trop. Hyg. Vol. 18, Beiheft 1, pp. 155-170, 1 fig. 1 est.

Trichomonas aus der Leber der Tauben. Cbl. f. Bakteriologie. Orig. P. 1. Vol. 71, pp. 184-189. Budapest. Ueber Infusoriendiarrhoe. Deutsch. Arch. f. Klin. Medizin Vol. 51, pp. 5:55-526.

1905 Die im menschlichen Darme vorkommenden Protozoen u. ihre Bedentung. Med. Klinik. T. 1 de 1905, pp. 13281331, 6 fig. Berlin.

Ueber die Bedeutung der Flagellaten im Magen u. Darm des Menschen. Deutsch. med. Wochenschr. Vol. 30, pp. 1717-1720 Leipzig.

1908 Sur un nouveau flagellé parasite de l'intestin des muscides au Congo Français. Compt. rend. de la Soc. de Biol. An. 60, t. 1, pp. 1106-1108, 1 fig.

ue des maladies des organes séxuels de la femme. Trad. franc. par les Drs. H. Dor et A. Socin. Paris. Cf. pp. 451-452.

Quelques remarques sur le Trichomonas vaginal de Donné. Compt. rend. de l'Acad. des Sc. Vol. 40, pp. 1076. 1077. Paris.

Ueber parasitäre Protozoan (Trichomonas pulmonalis) im Auswurf. Münch. med. Wochenschr. N. 51 de 1895, pp. 1181-1182, 1 fig. Bonn.

\section{Vol. 23, pp. 457-468.}

1895 Ueber des Vorkommen von Flagellaten im Darmkanal des Menschen. Cbl. f. Bakteriologie. Vol. 18, pp. 324327, 9 fig. Hannover. 1, T. I Fasc. 1, pp. 93-192.

Der Organismus der Infusionsthiere. III Der Organismus der Flagellaten oder Geisselinfusorien. Leipzig. 
STOKES, ALFRED C. 1884 Notices of some new parasitic protozoa. American naturalist. N. 18, pp. 1081-1086. Cf. 1082-1085. Philadelphia.

TERRY, BENJAMIN F. 1905 Two cases of chronic diarrhea with Trichomonas intestinalis in the stoals. Trans. of the Chicago path. Soc. Vol. 6, pp. 328-331.

UCKE, A.

WEISSENBERO

WENYON, C. M.

WENYON, C. M.

WENYON, C. M.

WIETING, J.

WOODCOCK, H. M. a. LAPAGE, G. ZABFL, ERICH
1907 Trichomonaden u. Megastomen im Menschendarm. Cbl. f. Bakteriologie. Vol. 45, pp. 231-232, 6 fig. St. Petersburg.

1912 Callimastix, gen. n. (perharps near Lophomonadina) cyclopts, sp. n. Ocurrence a morphology. Sitzungsber. der Geselsch. Naturforsch. Freunde. N. 5, pp. 299305, 1 fig.

1907 Observations on the protozoa in the intestine of mice. Arch. f. Protistenkunde. Suplem. 1, pp. 169-201 Cf. pp. 184-195, 3 est., 1 fig.

1910 A flagellate of the genus Cercomonas. Quart. J. of microsc. Science. Vol. 55, pp. 241-260, 19 fig.

1910 A new flagellate (Macrostoma Mesnili, n. sp.) from the human intestine with some remarks on the supposed cysts of Trichomonas. Parasitology. Vol. 3, pp. 210-216, 1 est., 2 fig.

1897 Ueber Flagellaten (Trichomonas) in der Lunge eines Schweines bei lobulären Pneumonie. Cbl. f. Bakteriologie. Orig. P. 1. Vol.2 1. pp. 721-725. Marburg.

On a remarkable new tipe of protistan parasite. Quart. J. of microsc. Science. Vol. 59, pp. 431-457, 2 est.

1910 Eiterüberschwemmung des Magendarmkanals aus Nasennebenhöhlenempiemem, nebst einer Bedeutung des Flagellaten befundes im Magen. Deutsch. med. Wochenschr. An. 36, pp. 798-800. 
Esplicação das estampas 1 e 2.

Figura 1 Sphaeromonas communis.

2 Sphaeromonas libetanzi.

3 Callimastix frontalis.

4 Chilomastix bittencourti.

5 Fórma atipica de Chilomastix caprae.

6 Fórma habitual de $C$. caprae.

7 Estadio muito adiantado de divisão binaria de $C$. caprae.

8 Chilomastix intestinalis.

9 Chilomastix cuniculi.

10 Chilomastix mesnili.

11 Chilomitus caviae; nucleo não visivel.

12 C. caviae; nucleo visivel, flajelos não visiveis.

13 Enteromonas hominis; flajelos em sua posição habitual.

$14 E$. hominis; aparelho nucleoflajelar completo; flajelos em posição anormal.

15 e 16 Fórmas de $E$. hominis sem flajelos visiveis.

17 Trichomastix caviae; axostilo não visivel.

18 Trichomonas caliae; nucleo sob a fórma de granulações chromofilas, das quaes uma maior parece representar o cariosoma.

19 Trichomonas muris; nucleo in- dividualizado; corpusculo chromatoide presente.

Figura 20 Trichomonas hominis; costa da membrana ondulante não distinta das granulações chromaticas proximas.

21 Trichomonas tatusi; axostilo não visivel.

22 Octomitns muris.

23 Giardia cuniculi; cariosomas contendo tres granulações chromaticas cada um e ligados aos corpusculos basaes por meio de rhizoplastos.

24 Giardia intestinalis; cariosomas pequenos; chromidio alongado, um pouco lateral e obliquamente dirigido; borda posterior chromofiia da ventosa, visivel.

$25 G$. intestinalis, de perfil; rizostilo e rizoplastos representados por duas linhas chromaticas paralelas; vê-se um dos nucleos com seu cariosoma.

26 Giardia muris; nucleos sem cariosoma e com uma granillação em crescente em seu polo posterior.

27 Fórma multiflajelada de Selenomonas ruminantium; fórma do corpo e disposição da chromatina normaes. 


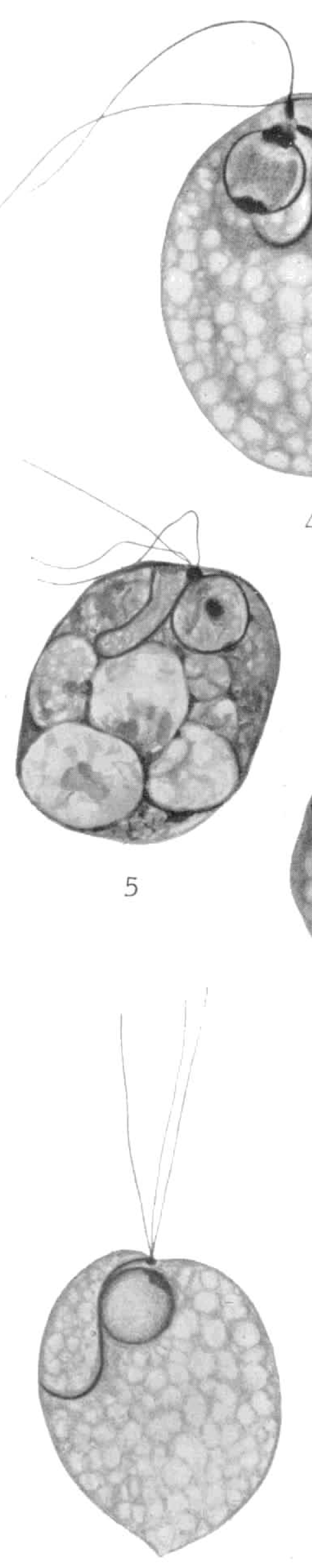

10
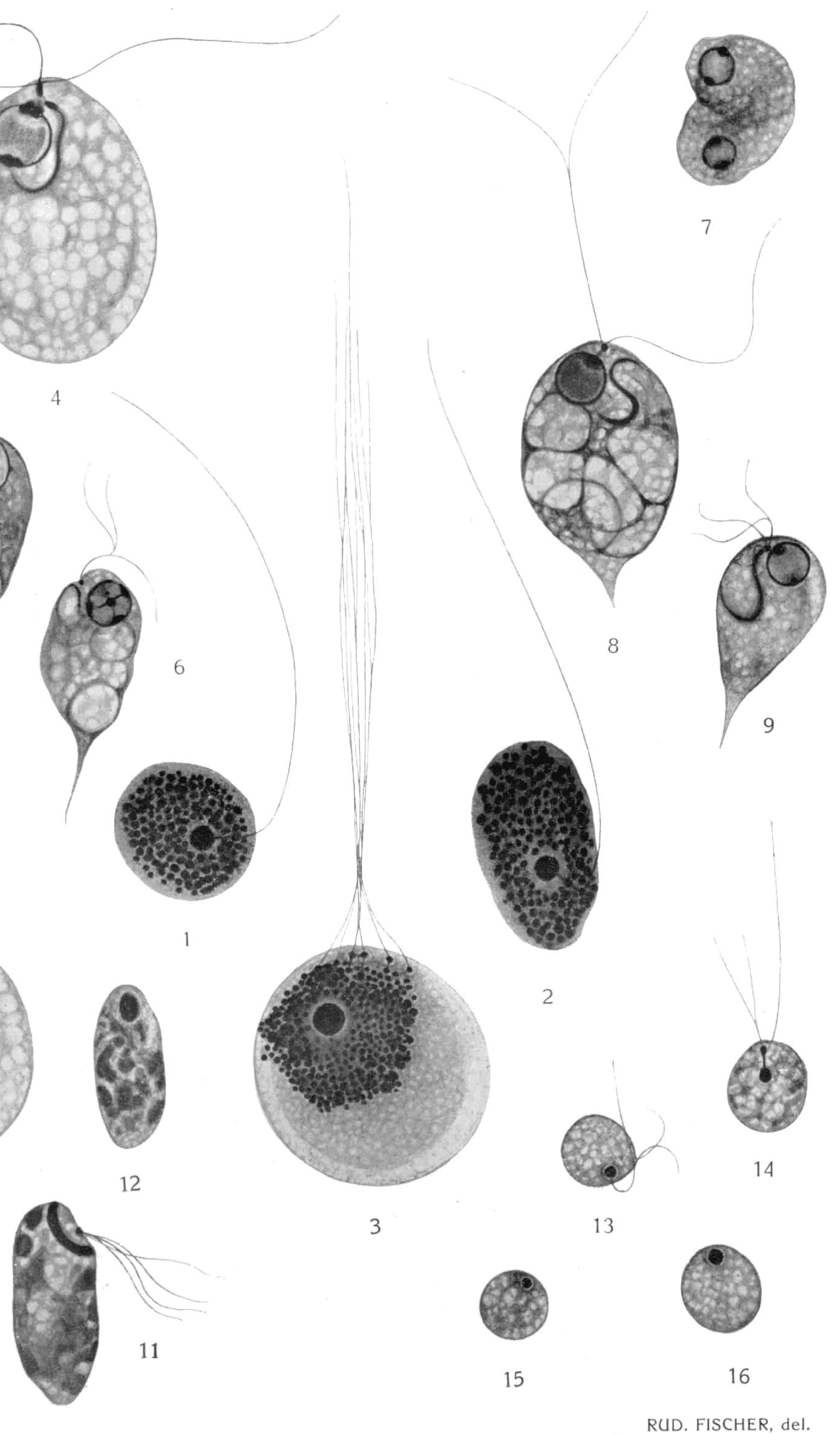


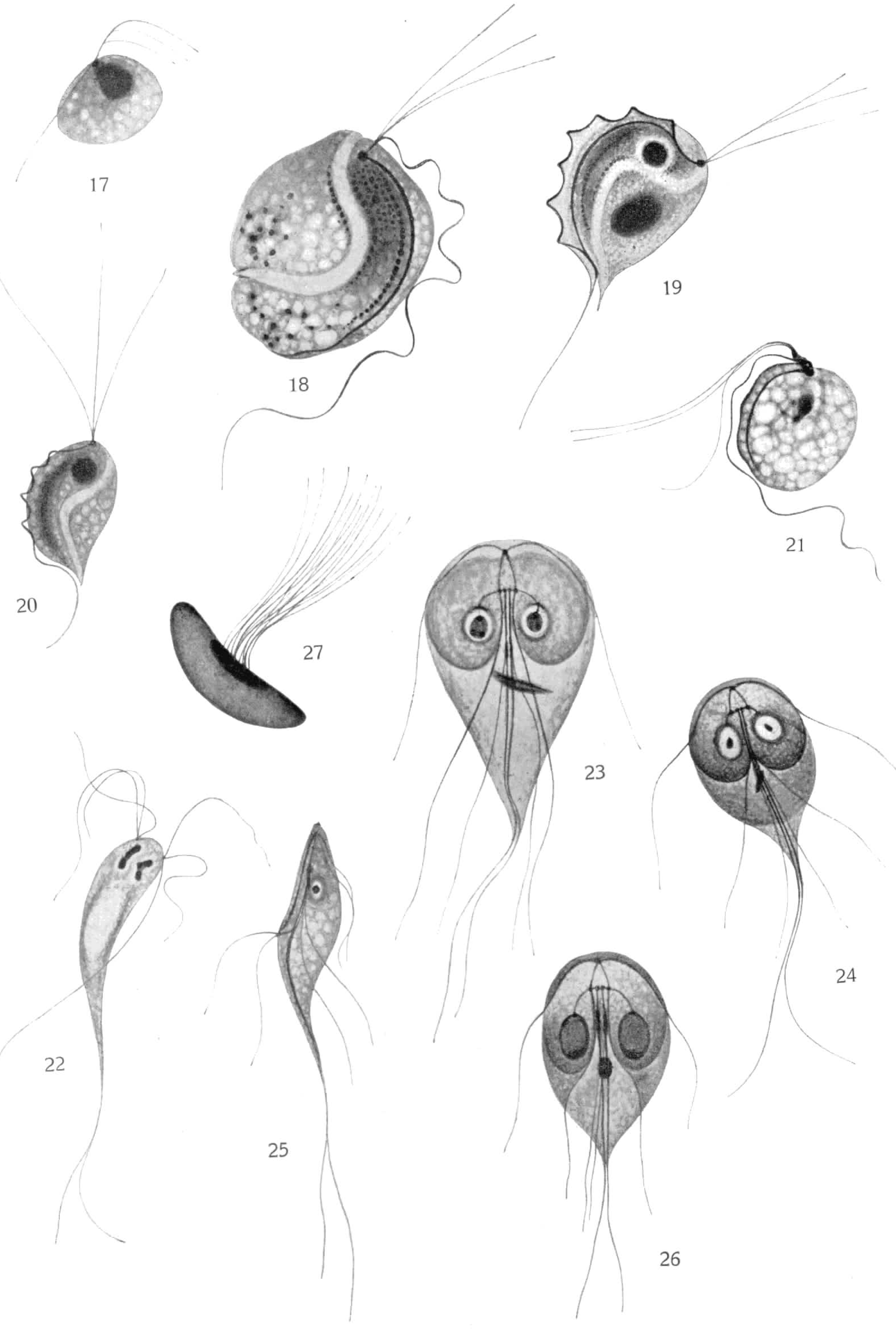

RUD. FISCHER, del. 